\title{
Review Article \\ Fiber-Wireless (FiWi) Broadband Access Networks in an Age of Convergence: Past, Present, and Future
}

\author{
Martin Maier \\ Optical Zeitgeist Laboratory, Institut National de la Recherche Scientifique (INRS), Montréal, QC, Canada H5A 1K6 \\ Correspondence should be addressed to Martin Maier; maier@ieee.org
}

Received 2 December 2013; Accepted 26 May 2014; Published 22 June 2014

Academic Editor: Roberto Proietti

Copyright (C) 2014 Martin Maier. This is an open access article distributed under the Creative Commons Attribution License, which permits unrestricted use, distribution, and reproduction in any medium, provided the original work is properly cited.

\begin{abstract}
After describing the beginnings and state of the art of integrated fiber-wireless (FiWi) broadband access networks in great detail, we briefly review recent progress and point to various ongoing research activities, including the design of energy-efficient "green" FiWi access networks, advanced survivability techniques, and integration of wireless and fiber optic sensors, towards realizing adaptable, dependable, and ecoconscious future-proof broadband access networks based on both wireless and shared passive fiber media. Furthermore, we discuss service, application, business, and operation related aspects, which motivate access technology to move into a substantially different direction in the long run than continued capacity provisioning. Given that most $4 \mathrm{G}$ cellular mobile network researches so far have been focusing on the achievable performance gains in the wireless front-end only without looking into the details of backhaul implementations and possible backhaul bottlenecks, we identify open key research challenges for FiWi broadband access networks. We explore ways of how they can be deployed across relevant economic sectors other than telecommunications per se, taking major paradigm shifts such as the Third Industrial Revolution, Energy Internet, smart grid, and explosion of mobile data traffic in today's cellular networks into account.
\end{abstract}

\section{Introduction}

According to the Federal Communications Commission (FCC), broadband enables individuals and enterprises to access a wide range of resources, services, and products related to education, culture, entertainment, telemedicine, ecommerce, public safety, and homeland security. In a detailed study carried out by the Organisation for Economic Cooperation and Development (OECD) [1] it was shown that the impact of providing residential and business subscribers with broadband access is manifold. Among others, broadband enables the emergence of business models, processes, and inventions as well as improved goods and services. Furthermore, broadband increases competitiveness and flexibility in the economy by the increased diffusion of information at lower cost and by improving market access to increasingly larger markets.

Figure 1(a) shows the fixed wired and wireless access technologies used by broadband subscribers in 2010. Back then, the majority of fixed broadband subscribers deployed digital subscriber line (DSL) or cable modem, while only $9 \%$ of the 271 million subscribers were connected via fiber-tothe-premises solutions, that is, fiber-to-the-home (FTTH) or fiber-to-the-building (FTTB) with local area network (LAN) deployment in apartments. (For completeness, we note that the remaining $2 \%$ of subscribers were provided broadband access via satellite, WiMAX, or broadband over power line (BPL).) However, this situation is changing rapidly. For illustration, Figure 1(b) shows the latest OECD data on fixed wired broadband subscriptions as of June 2012. Clearly, this figure illustrates that an increasing percentage of broadband subscribers rely on fiber access technologies at the expense of legacy DSL solutions. This trend is expected to become even more pronounced over the next couple of decades. In the short- to midterm, current state-of-the-art very high bit-rate DSL (VDSL) may be superseded by next-generation copper based solutions supported by deep fiber access networks getting increasingly closer to subscribers.

We are currently witnessing a strong worldwide deployment of deep fiber access solutions to push optical fiber closer to individual homes and businesses and to help realize FTTx networks, whereby $\mathrm{x}$ denotes the discontinuity between 


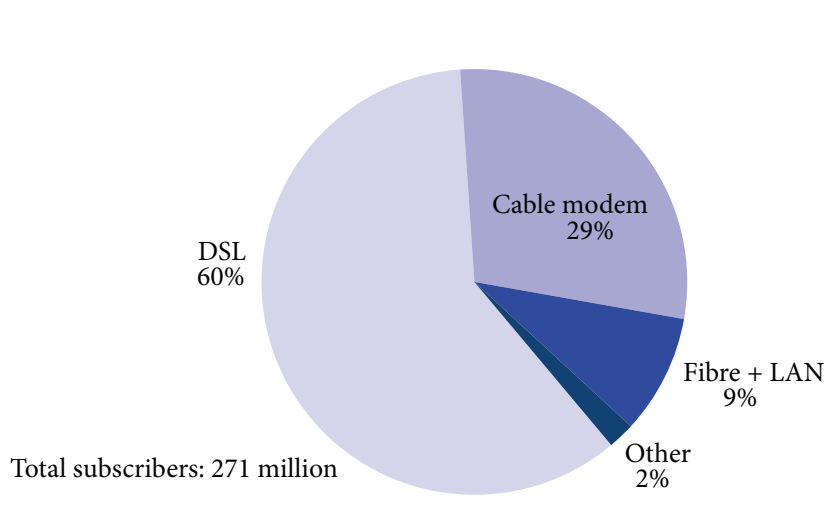

(a)

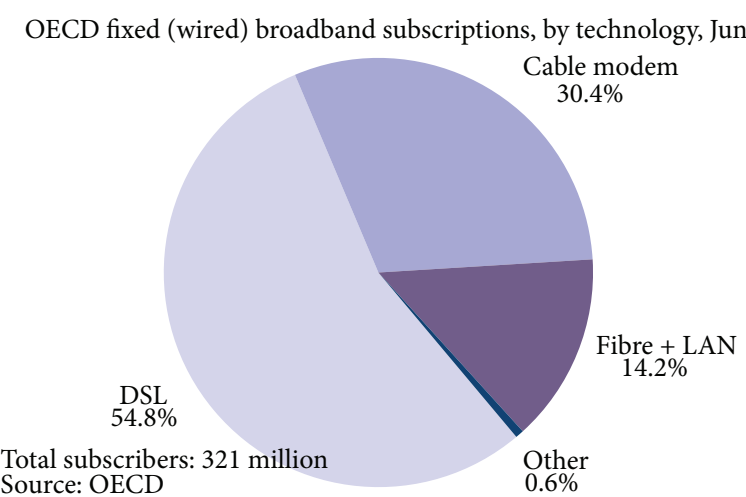

(b)

FIGURE 1: Broadband subscribers across OECD countries by technology: (a) fixed wired and wireless access technologies used in 2010 and (b) fixed wired access technologies used in 2012 [2].

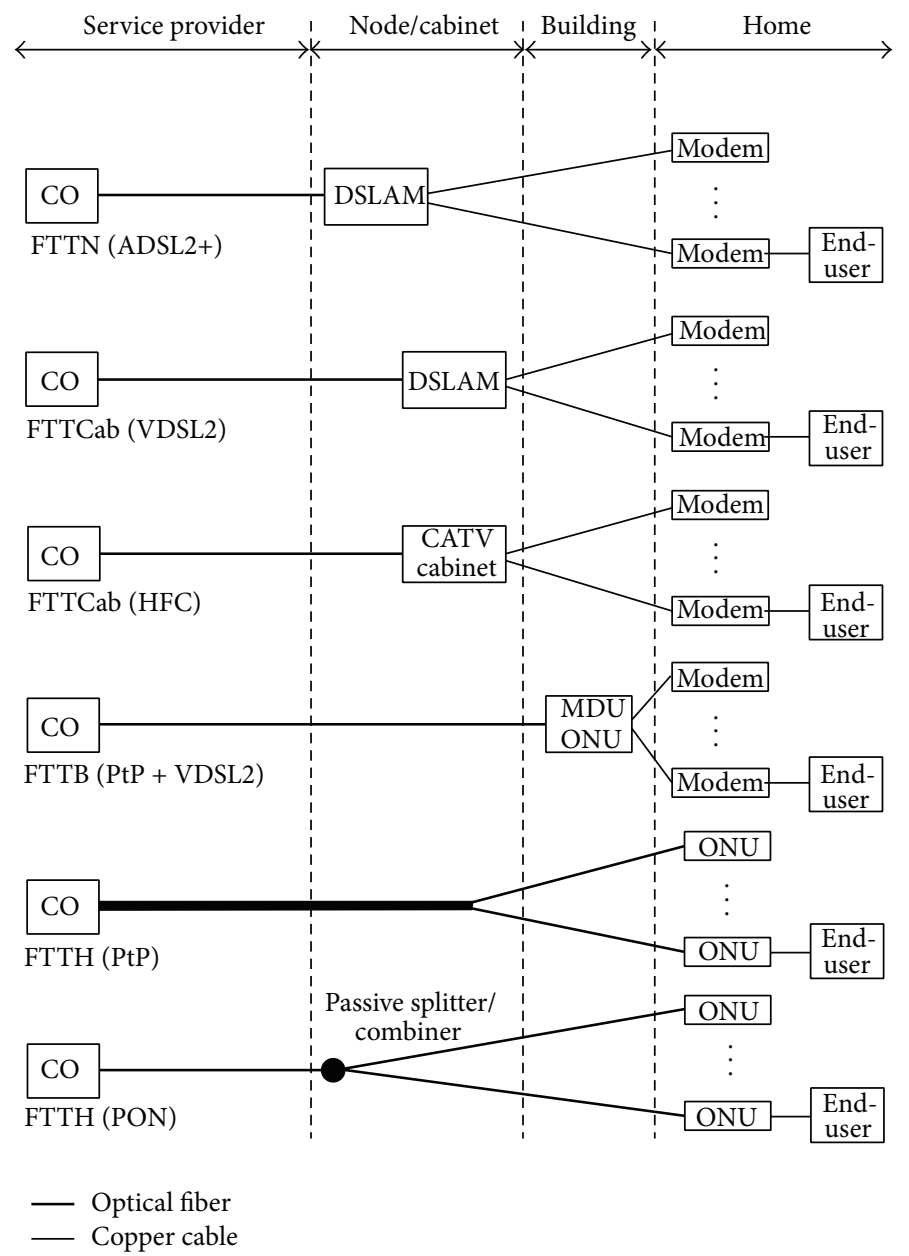

FIGURE 2: FTTx network architectures.

optical fiber and some other transmission medium [3]. Figure 2 shows a variety of possible FTTx network architectures, ranging from fiber-to-the-node (FTTN) to FTTH networks, depending on how deep optical fiber reaches into the first/last mile. In FTTN networks, fiber is used between the central office (CO) and the DSL access multiplexer (DSLAM) located at the remote node. The DSLAM connects to each end-user's modem through a separate legacy telephone twisted pair of copper wires. FTTN networks deploy asymmetric DSL2+ (ADSL2+) on the twisted pairs offering 
data rates of up to $24 \mathrm{Mb} / \mathrm{s}$ downstream and up to $3.5 \mathrm{Mb} / \mathrm{s}$ upstream. In fiber-to-the-cabinet (FTTCab) networks, also known as fiber-to-the-curb (FTTC) networks, the DSLAM is moved closer to the end-user in order to shorten the length of the twisted-pair drop lines and thereby enable the deployment of VDSL, which offers significantly increased data rates of up to $52 \mathrm{Mb} / \mathrm{s}$ downstream and up to $16 \mathrm{Mb} / \mathrm{s}$ upstream. FTTCab is also used by cable network operators to build hybrid fiber-coax (HFC) networks, where the drop lines are realized through coax cables instead of twisted pairs. Bringing fiber all the way to buildings gives rise to FTTB networks. Typically, FTTB networks use a multidwelling unit (MDU) optical network unit (ONU) in the basement of the building to terminate the optical signal coming from the $\mathrm{CO}$ and distribute the converted signal across a separate network inside the building. Finally, paving all the way to the home with optical fiber leads to FTTH networks, which come in two flavors: (i) point-to-point (PtP) star topology, where each ONU is connected to the CO via a separate fiber or (ii) pointto-multipoint passive optical network (PON) tree topology, using a single shared fiber link between the $\mathrm{CO}$ and a passive optical splitter/combiner at the remote node.

Deep fiber access is a challenging mix of technology choices, business models, and regulatory issues. But due to increasingly personalized, interactive, on-demand, and highdefinition broadband service demands and thanks to innovations within low-cost fiber-laying techniques, for example, microtrenching, there is no doubt that widespread FTTH/B deployment is just a matter of time [4]. According to recent market data by ABI Research, the number of fixed broadband subscribers will rise to 501 million by the end of 2014, of which 106 million will subscribe to services delivered via fiber. Among the three major fixed broadband technologies, the number of fiber subscribers is increasing fastest at a compound annual growth rate of 20\% from 2008 to 2014 . Furthermore, FCC has recently unveiled the United States' first national broadband plan with the goal to provide every American with broadband access speeds of $100 \mathrm{Mb} / \mathrm{s}$ by 2020. Toward this goal, fiber (together with next-generation wireless) broadband technologies will play an increasingly vital role in future broadband access networks. This is already witnessed by installed state-of-the-art VDSL equipment, which is almost exclusively based on optical fiber backhaul solutions. While copper will certainly continue to play an important role in current and near-term broadband access networks, it is expected that FTTH deployment volume will keep increasing gradually and will eventually become the predominant fixed wireline broadband technology by 2035 [5].

Optical fiber provides an unprecedented bandwidth potential that is far in excess of any other known transmission medium and offers significantly longer ranges without requiring any active devices. Optical fiber has some further advantageous properties such as longevity and low maintenance costs, which will eventually render fiber the medium of choice in wired first/last mile access networks. In fact, this trend can already be observed in most of today's greenfield deployments where fiber rather than copper cables is installed for broadband access [6]. Optical access networks provide transparency against data rate and signal format, which eased carriers worldwide into deploying future-proof PON outside plants that can be flexibly upgraded as new technologies mature or new standards evolve [7]. Arguing that due to its unique properties optical fiber is likely to entirely replace copper wires in the near-to midterm, we will elaborate on the final frontier of optical networks, namely, the convergence with their wireless counterparts. Optical and wireless technologies can be thought of as quite complementary and will expectedly coexist over the next decades. Future broadband access networks will be bimodal, capitalizing on the respective strengths of both technologies and smartly merging them in order to realize future-proof fiber-wireless (FiWi) networks that strengthen our information society while avoiding its digital divide. By combining the capacity of optical fiber networks with the ubiquity and mobility of wireless networks, FiWi networks form a powerful platform for the support and creation of emerging as well as future unforeseen applications and services, for example, telepresence [8].

In this paper, we provide a comprehensive overview of the beginnings, state of the art, and latest developments of FiWi broadband access networks. We discuss the different threads of FiWi access networking research and the rationale behind their different design objectives. After describing recent progress, we elaborate on the role of FiWi access networks in the dawning age of convergence and outline some exciting research directions for future FiWi access networks. The remainder of this paper is structured as follows. Section 2 describes related research topics and defines FiWi access networks as a new research area. In Section 3, we review the state of the art of FiWi broadband access networks, while recent progress is described in Section 4. Section 5 provides an outlook and outlines the road ahead for future FiWi access networks. Finally, we draw some conclusions in Section 6.

\section{FiWi Broadband Access Networks}

Traditionally, wireless and optical fiber networks have been designed separately from each other. Wireless networks aimed at meeting specific service requirements while coping with particular transmission impairments and optimizing the utilization of the system resources to ensure cost-effectiveness and satisfaction for the user. In optical networks, on the other hand, research efforts rather focused on cost reduction, simplicity, and future proofness against legacy and emerging services and applications by means of optical transparency. Wireless and optical access networks can be thought of as complementary. Optical fiber does not go everywhere, but where it does go, it provides a huge amount of available bandwidth. Wireless access networks, on the other hand, potentially go almost everywhere but provide a highly bandwidth-constrained transmission channel susceptible to a variety of impairments.

Future broadband access networks not only have to provide access to information when we need it, where we need it, and in whatever format we need it, but also, and arguably more importantly, have to bridge the digital divide and offer simplicity and user-friendliness based on open standards in order to stimulate the design of new applications and 
services. Toward this end, future broadband access networks must leverage on both optical and wireless technologies and converge them seamlessly, giving rise to FiWi access networks [9]. FiWi access networks are instrumental in strengthening our information society while avoiding its digital divide. By combining the capacity of optical fiber networks with the ubiquity and mobility of wireless networks, FiWi networks form a powerful platform for the support and creation of emerging as well as future unforeseen applications and services. FiWi networks hold great promise to change the way we live and work by replacing commuting with teleworking. This not only provides more time for professional and personal activities for corporate and our own personal benefit but also helps reduce fuel consumption and protect the environment; issues that are becoming increasingly important in our lives.

Due to the difficulty and prohibitive costs of supplying optical fiber to all end-user premises as well as the spectrum limitations of wireless access networks, bimodal FiWi access networks are more attractive than relying on either standalone access solution. FiWi access networks are realized by integrating wireless access technologies, for example, cellular, WiMAX, and WiFi, with installed optical fiber infrastructure that has been pushed ever closer toward end-users over the last few years and has been the preferred medium of choice in most of today's greenfield deployments, where fiber rather than copper cables are installed for broadband access. To better understand the rationale behind the vision of FiWi access networks, we first describe related research topics and then define FiWi access networks as a new research area in the following.

Fixed Mobile Convergence (FMC). According to the European Telecommunications Standardization Institute (ETSI), FMC is concerned with developing network capabilities and supporting standards that may be used to seamlessly offer a set of consistent services via fixed or mobile access to fixed or mobile, public or private networks, independently of the access technique [10].

FMC can be done at different levels, for example, business or service provisioning level. Note, however, that FMC does not necessarily imply the physical convergence of networks. In fact, the convergence at the network facilities level, where an operator uses the same physical network infrastructure with common transmission and switching systems to provide both mobile and fixed services, is more accurately referred to as fixed mobile integration (FMI) [11]. While FMI considers any type of access networks, for example, DSL, HFC, or wireless local area network (WLAN), the so-called optical wireless integration (OWI) focuses on emerging optical and wireless broadband access technologies, as explained next.

Optical Wireless Integration (OWI). Current copper based access network technologies such as DSL and HFC face serious challenges to meet the requirements of future broadband access networks. While DSL suffers from severe distance and noise limitations, HFC falls short to efficiently carry data traffic due to its upstream noise and crosstalk accumulation. Recent progress in optical fiber technologies, especially the maturity of integration and new packaging technologies, has rendered optical fiber access networks a promising low-cost broadband solution. In particular, PONs are able to provide lower network deployment and maintenance costs as well as longer distances than current DSL and HFC networks. OWI aims at integrating PONs and other optical fiber access technologies with broadband wireless access technologies, for example, WiMAX, in order to increase the capacity of wireless access networks and reduce access point complexity through centralized management [12].

It is important to note that there is a difference between OWI and free-space optical wireless (OW) communications. OW systems to test line-of-sight wireless communications were already designed in the 1960's, well before the development of optical fiber communications [13]. Current radio frequency (RF) wireless systems, for example, WiFi, WiMAX, or ultrawideband (UWB), are limited in both data rate and range due to their low carrier frequencies. OW communications links operate at much higher carrier frequencies than their RF counterparts. As the RF spectrum becomes increasingly congested, OW represents an interesting alternative to RF wireless systems. OW may be deployed as a temporary backbone for rapidly deployable mobile wireless communication infrastructure, especially in densely populated urban areas. Note, however, that unlike OWI networks, OW links and networks do not involve any wired (fiber) infrastructure.

Radio-over-Fiber (RoF) versus Radio-and-Fiber (R\&F) Networks. RoF networks have been studied for many years as an approach to integrate optical fiber and wireless networks. In RoF networks, RFs are carried over optical fiber links between the $\mathrm{CO}$ and multiple low-cost remote antenna units (RAUs) in support of a variety of wireless applications, for example, microcellular radio systems [14]. It was experimentally demonstrated that RoF networks can have an optical fiber range of up to $50 \mathrm{~km}$. However, inserting an optical distribution system in wireless networks may have a major impact on the performance of medium access control (MAC) protocols [15]. The limitations of RoF networks can be avoided in so-called radio-and-fiber (R\&F) networks [16]. While RoF networks use optical fiber as an analog transmission medium between the $\mathrm{CO}$ and one or more RAUs with the $\mathrm{CO}$ being in charge of controlling access to both optical and wireless media, in R\&F networks access to the optical and wireless media is controlled separately from each other by using in general two different MAC protocols in the optical and wireless media, with protocol translation taking place at their interface. As a consequence, wireless MAC frames do not have to travel along the optical fiber to be processed at the $\mathrm{CO}$ but simply traverse their associated wireless access point and remain in the wireless front-end, thus avoiding the negative impact of fiber propagation delay on the network performance.

FiWi Access Networks. FiWi access networks may deploy both RoF and R\&F technologies. By simultaneously providing wired and wireless services over the same infrastructure, FiWi access networks are able to consolidate optical and wireless access networks that are usually run independently of each other, thus potentially leading to major cost savings. 
FiWi networking research deals with the OWI of optical and wireless broadband access technologies, for example, wireless mesh network (WMN). FiWi research focuses on the physical (PHY), MAC, and network layers with the goal to develop and investigate low-cost enabling FiWi technologies as well as layer-2 and layer-3 protocols and algorithms. Higher-layer network capabilities developed through FMC standardization efforts can be exploited on top of the PHY, MAC, and network layers of FiWi networks. FiWi research inquires new methods of optical RF generation exploiting fiber nonlinearities and various modulation techniques. Specifically, to avoid the electronic bottleneck, the generation of RF signals is best done optically. A variety of different optical RF generation techniques were experimentally studied and demonstrated, including four-wave mixing (FWM) or cross-phase modulation (XPM) in a highly nonlinear dispersion-shifted fiber (HNL-DSF), cross-absorption modulation (XAM) in an electroabsorption modulator (EAM), and external intensity modulation (IM) or phase modulation (PM) [17]. According to [17], external intensity and phase modulation schemes are the most practical solutions for all-optical RF generation due to their low cost, simplicity, and long-distance transmission performance. Furthermore, FiWi research also includes the study of different remodulation schemes for the design of colorless (i.e., wavelengthindependent) RAUs. Remodulation techniques also play a key role in ongoing research activities on full-duplex wavelength division multiplexing (WDM) PONs based on colorless ONUs. Remodulation capitalizes on a single light source at the central office to generate a downlink wavelength that is reused at RAUs/ONUs for upstream transmission, thereby avoiding the need for an additional light source at each RAU/ONU. A number of different remodulation schemes have been proposed and investigated, for example, differentiated phase-shift keying (DPSK) for downstream and onoff-keying (OOK) for upstream, optical carrier suppression (OCS) for downstream and reused for upstream, or PM for downstream and directly modulated semiconductor optical amplifier (SOA) for upstream. The use of a colorless SOA as an amplifier and modulator for upstream transmission provides a promising low-cost RoF solution that is easy to maintain [17].

While significant progress has been made at the PHY layer of FiWi and in particular RoF transmission systems, FiWi networking research on layer- 2 and layer-3 related issues has begun only recently. Among others, FiWi layer$2 / 3$ research includes the joint optimization of performanceenhancing MAC mechanisms separately used in the wireless and optical network segments, for example, wireless frame aggregation and optical burst assembly, hybrid access control protocols, integrated path selection algorithms, and advanced resilience techniques. Layer-2/3 networking research is crucial to unleash the full potential of FiWi broadband access networks $[18,19]$.

\section{State of the Art}

Beside cell-based RoF networks, a number of FiWi network architectures were proposed, which can be classified based on their wireless access technologies: WiMAX or WiFi. As we will see shortly, different challenges were addressed such as routing and wireless channel assignment, which can be performed completely either in the wireless domain by the base station (BS) or access point (AP), or by an optical network element, for example, CO or optical line terminal (OLT). The level of provided quality-of-service (QoS) largely depends on the performance of the implemented routing and resource management algorithms, including bandwidth allocation and channel assignment algorithms with absolute or relative QoS assurances. Reconfiguration is another previously addressed challenging issue that involves resource management in the wireless and/or optical part.

\subsection{Architectures}

3.1.1. Cellular Architectures. Cellular networks used for fast moving users, for example, train passengers, suffer from frequent handovers when hopping from one BS to another one. The frequent handovers may cause numerous packet losses, resulting in a significantly decreased network throughput. An interesting approach to solve this problem is the use of an RoF network installed along the rail tracks in combination with the so-called moving cell concept [20].

Recently, the moving extended cell concept was proposed to provide connectivity for any possible direction [21]. The fiber optic network becomes a means for speedy handoff between base stations that serve the mobile users. A hybrid frequency division multiplexing (FDM)/WDM network architecture was used to support the delivery of multiple RF channels in the $60 \mathrm{GHz}$ frequency band over the same wavelength. The extended cell involves the current user's cell and the surrounding cells ensuring connectivity for any random direction. The extended cell is adaptively restructured when the user enters a new cell. It was shown that the proposed concept can provide zero packet loss and call dropping probability in high-rate wireless services for a wide range of mobile speeds of up to $40 \mathrm{~m} / \mathrm{sec}$, independently of the fiber link distances.

RoF networks are attractive since they provide transparency against modulation techniques and are able to support various digital formats and wireless standards in a costeffective manner. While single-mode fibers (SMFs) are typically found in outdoor optical networks, many buildings have preinstalled multimode fiber (MMF) cables. Cost-effective MMF-based networks can be realized by deploying low-cost vertical cavity surface emitting lasers (VCSELs). Apart from realizing low-cost microcellular radio networks, optical fibers can also be used to support a wide variety of other radio signals. In [22], a low-cost MMF network was experimentally tested to demonstrate the feasibility of indoor radio-overMMF networks for the in-building coverage of secondgeneration and third-generation cellular radio networks as well as IEEE $802.11 \mathrm{~b} / \mathrm{g}$ WLAN.

3.1.2. WiMAX-Based Architectures. The integration of Ethernet PON (EPON) and WiMAX access networks can be done in several ways. According to [23], the following four architectures can be used. 
Independent Architecture. In this approach, WiMAX base stations serving mobile client nodes are attached to an ONUthe customer premises equipment (CPE) of an EPONjust like any other wired subscriber node. WiMAX and EPON networks are connected via a common standardized interface, for example, Ethernet, and operate independently from each other.

Hybrid Architecture. This approach introduces an ONUbase station (ONU-BS) that integrates the EPON ONU and WiMAX BS in both hardware and software. The integrated ONU-BS controls the dynamic bandwidth allocation of both ONU and BS.

Unified Connection-Oriented Architecture. Similar to the hybrid architecture, this approach deploys an integrated ONU-BS. But instead of carrying Ethernet frames, WiMAX MAC protocol data units (PDUs) containing multiple encapsulated Ethernet frames are used. By carrying WiMAX MAC PDUs, the unified architecture can be run like a WiMAX network with the ability to grant bandwidth finely using WiMAX's connection-oriented rather than EPON's queueoriented bandwidth allocation.

Microwave-over-Fiber Architecture. In this approach, the WiMAX signal is modulated on a wireless carrier frequency and is then multiplexed and modulated together with the baseband EPON signal onto a common optical frequency (wavelength) at the ONU-BS. The central node consists of a conventional EPON OLT and a central WiMAX BS, called macro-BS. The OLT processes the baseband EPON signal, while the macro-BS processes data packets originating from multiple WiMAX BS units.

3.1.3. WiFi-Based Architectures. Besides the aforementioned integration approaches of EPON and WiMAX networks, several other FiWi architectures based on WiFi technology have been studied, as described in the following.

Unidirectional Ring. This type of FiWi network architecture interconnects the $\mathrm{CO}$ with multiple WiFi-based wireless APs by means of an optical unidirectional fiber ring [24]. The CO is responsible for managing the transmission of information between mobile client nodes (MCNs) and their associated APs as well as acting as a gateway to other networks. Each AP provides wireless access to MCNs within its range. All MCNs take part in the topology discovery, whereby each MCN periodically sends the information about the beacon power received from its neighbors to its associated AP. In doing so, APs are able to estimate the distances between MCNs and compute routes. Multihop relaying is used to extend the range. To enhance the reliability of the wireless link, the CO sends information to two different APs (path diversity). The proposed implementation can support advanced path diversity techniques that use a combination of transmission via several APs and multihop relaying, for example, cooperative diversity or multihop diversity. Consequently, the CO must be able to assign channels quickly and efficiently by using one or more wavelength channels on the fiber ring to accommodate multiple services such as WLAN and cellular radio network.

Bidirectional Ring. This WiFi-based architecture consists of a two-level bidirectional path-protected ring (BPR) architecture for dense WDM (DWDM)/subcarrier multiplexing (SCM) broadband FiWi networks [25]. In this architecture, the $\mathrm{CO}$ interconnects remote nodes (RNs) via a dual-fiber ring. Each RN cascades APs through concentration nodes (CNs), where each AP offers services to MCNs. For protection, the $\mathrm{CO}$ is equipped with two sets of devices (normal and standby). Each RN consists of a protection unit and a bidirectional wavelength add-drop multiplexer based on a multilayer dielectric interference filter. Each $\mathrm{CN}$ contains a protection unit. The AP comprises an optical transceiver, a protection unit, up/down RF converters, and a sleeve antenna. Each AP provides channel bandwidth of at least $5 \mathrm{MHz}$ and covers up to $16 \mathrm{MCNs}$ by means of FDM. Under normal operating conditions, the CO transmits downstream signals in the counter-clockwise direction via RNs and CNs to the APs. If a fiber cut occurs between two RNs or between two CNs, their associated controllers detect the failure by monitoring the received optical signal and then switch to the clockwise protection ring. If a failure happens at an AP, the retransmitted signals are protection switched through other optical paths by throwing an optical switch inside the affected AP. This architecture provides high reliability, flexibility, capacity, and self-healing properties.

Hybrid Star-Ring. This hybrid FiWi architecture combines optical star and ring networks [26]. Each fiber ring accommodates several WiFi-based APs and is connected to the CO and two neighboring fiber rings via optical switches. The optical switches have full wavelength conversion capability and interconnect the APs and CO by means of shared pointto-point lightpaths. The network is periodically monitored during prespecified intervals. At the end of each interval, the lightpaths may be dynamically reconfigured in response to varying traffic demands. When traffic increases and the utilization of the established lightpaths is low, the load on the existing lightpaths is increased by means of load balancing. Otherwise, if the established lightpaths are heavily loaded, new lightpaths need to be set up, provided enough capacity is available on the fiber links. In the event of one or more link failures, the affected lightpaths are dynamically reconfigured using the redundant fiber paths of the architecture.

Unidirectional Ring-PON. This FiWi network proposed in [27] consists of an optical WDM backhaul ring with multiple single-channel or multichannel PONs attached to it. More precisely, an optical add-drop multiplexer (OADM) is used to connect the OLT of each PON to the WDM ring. Wireless gateways are used to bridge PONs and WMN. In the downstream direction, data packets are routed from the $\mathrm{CO}$ to the wireless gateways through the optical backhaul and are then forwarded to the MCNs by wireless mesh routers. In the upstream direction, wireless mesh routers forward data packets to one of the wireless gateways, where they 


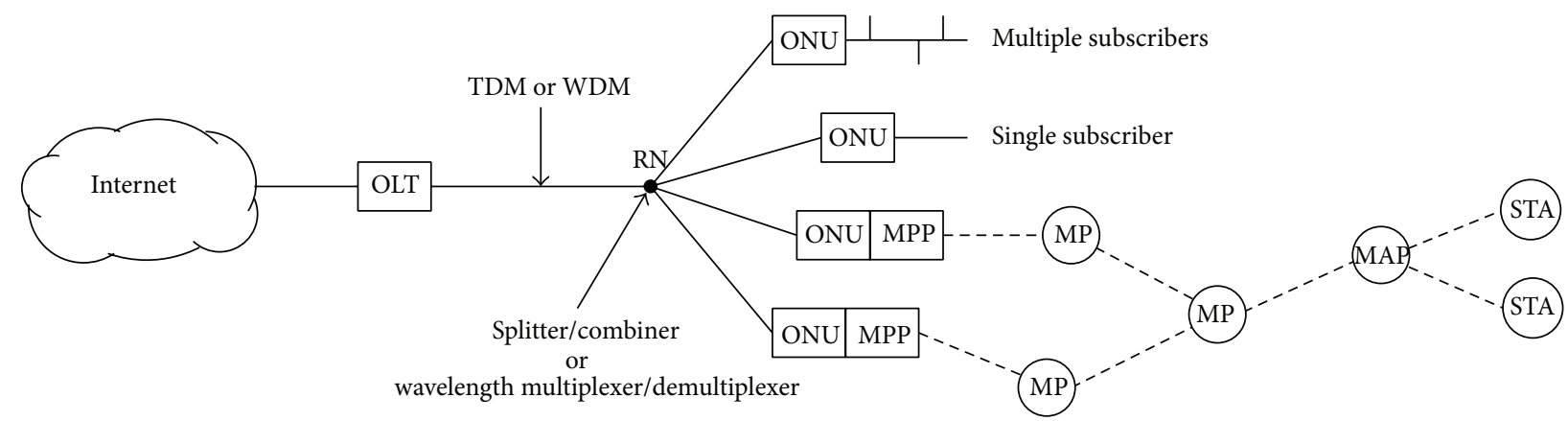

FIGURE 3: FiWi access network architecture based on single-stage TDM or WDM PON and multihop WMN using legacy or VHT WLAN technologies.

are then transmitted to the $\mathrm{CO}$ on one of the wavelength channels of the optical backhaul WDM ring, as each PON operates on a separate dynamically allocated wavelength channel. Since the optical backhaul and WMN use different technologies, an interface is defined between each ONU and the corresponding wireless gateway in order to monitor the WMN and perform route computation taking the state of wireless links and average traffic rates into account. When the traffic demands surpass the available PON capacity, some of the single-channel time division multiplexing (TDM) PONs may be upgraded to WDM PONs. If some PONs are heavily loaded and others have less traffic, some heavily loaded ONUs may be assigned to a lightly loaded PON by tuning their optical transceivers to the wavelength assigned to the lightly loaded PON. This architecture provides cost-effectiveness, bandwidth efficiency, wide coverage, high flexibility, and scalability. In addition, the reconfigurable TDM/WDM optical backhaul helps reduce network congestion and average packet latency by means of load balancing. Moreover, the dynamic allocation of radio resources enables cost-effective and simple handovers.

Cascaded PON-WMN. The majority of previous studies on FiWi access networks considered a cascaded architecture consisting of a single-stage PON and a multihop WMN, as shown in Figure 3. Typically, the PON under consideration is a conventional IEEE 802.3ah compliant wavelengthbroadcasting TDM EPON based on a wavelength splitter/combiner at the RN, using one time-shared wavelength channel for upstream transmissions (from ONUs to OLT) and another time-shared wavelength channel for downstream transmissions (from OLT to ONUs), both operating at a data rate of $1 \mathrm{~Gb} / \mathrm{s}$. A subset of ONUs may be located at the premises of residential or business subscribers, whereby each ONU provides FTTH/B services to a single or multiple attached wired subscribers. The remaining ONUs are assumed to be equipped with a mesh portal point (MPP) to interface with the WMN, which in turn consists of relay mesh points (MPs) and mesh access points (MAPs) to provide stations (STAs) with wireless access to the FiWi network. Most previous FiWi studies assumed a WMN based on IEEE 802.11a/b/g WLAN technologies, offering a maximum raw data rate of $54 \mathrm{Mb} / \mathrm{s}$ at the physical layer.
Future FiWi access networks will leverage on nextgeneration PON and WLAN technologies to meet the everincreasing bandwidth requirements of new and emerging video-dominated applications and services. A variety of nextgeneration $\mathrm{PON}$ technologies are currently investigated to enable short-term evolutionary and long-term revolutionary upgrades of coexistent Gigabit-class TDM PONs [28]. However, the most promising solutions for PON evolution toward higher bandwidth per user are (i) data rate upgrades to $10 \mathrm{~Gb} / \mathrm{s}$ and higher and (ii) multiwavelength channel migration toward wavelength-routing or wavelength-broadcasting WDM PONs with or without cascaded TDM PONs, respectively $[29,30]$. Similarly, to alleviate the bandwidth bottleneck of wireless mesh front-end networks, future FiWi access networks are expected to be based on next-generation IEEE 802.11n WLAN, which offers data rates of $100 \mathrm{Mb} / \mathrm{s}$ or higher at the MAC service access point, as well as emerging Gigabitclass IEEE 802.1lac very high throughput (VHT) WLAN technologies that achieve raw data rates of up to $6900 \mathrm{Mb} / \mathrm{s}$.

\subsubsection{Highlighted Architectures with a Physical Layer Focus}

GROW-Net. The so-called grid reconfigurable optical-wireless network (GROW-Net) is a scalable municipal FiWi network architecture that adapts the street layout in a city and makes use of available dark fibers readily available in urban areas $[31,32]$. The design objectives of GROW-Net are to provide scalability in terms of bandwidth and allow for infrastructure extensibility. The basic building block of GROW-Net is the grid-cell, which provides the backbone fiber connectivity to wireless gateways. The optical fiber backbone infrastructure is extensible using the proposed $\mathrm{H}$-tree partitioning process, whereby at each stage of the process the spacing between two adjacent WMN routers is reduced by $50 \%$ and optical terminals are inserted and interconnected by means of optical WDM links that are laid out in an H-shape. In the downstream direction, the central hub of GROW-Net deploys a tunable laser, which can reach different optical terminals by tuning the laser to the respective wavelength channel supported by the intended optical terminal. Each optical terminal is equipped with a thin film filter (TFF), which lets pass only the corresponding wavelength channel of a given optical terminal. In the upstream direction, each optical terminal is 
made colorless by deploying a reflective semiconductor optical amplifier (RSOA) for remote modulation of a continuous wavelength signal sent by the central hub. GROW-Net is able to achieve increased fiber protection than conventional treebased PONs due to its mesh topology.

FUTON. In the European research project FUTON (Fiber Optic Networks for Distributed, Extendible, Heterogeneous Radio Architectures and Service Provisioning), an integrated wireless/optical network architecture was developed that aims at enabling wireless services and meeting ITU's IMTAdvanced (IMT-A) requirements in a cost-efficient manner [33-35]. In the proposed architecture, low-complexity RAUs are connected via analog RoF links to a central processing unit (CU), which performs joint signal processing and resource management. Signals from the CU to different antennas of a site and to different RAUs can be multiplexed onto the same optical fiber by means of WDM and SCM. The architecture's ability was demonstrated by developing a FUTON prototype, which features downlink data transmission from two single-antenna RAUs to two single-antenna mobile terminals. To separate the users spatially, an MMSE precoding filter is applied at the CU. In the downlink, pilots and data are transmitted to the mobile terminals, whereas the uplink is used to feed channel state information from the mobile terminals back to the $\mathrm{CU}$ to adapt the precoding filter. The obtained measurements demonstrated that the MMSE precoding provides a large throughput gain compared to conventional noncooperative precoding. This is due to the fact that the MMSE precoding is able to mitigate the mutual interference of the RAUs such that both mobile terminals can receive data at high data rates using the same time and frequency resources, at the expense of a significant overhead in the uplink.

ACCORDANCE. Another interesting European research project is the so-called ACCORDANCE, an ultrahigh capacity extended reach optical access network architecture based on orthogonal frequency division multiple access (OFDMA) technology/protocols, implemented through the proper mix of state-of-the-art photonics and electronics [36-38]. ACCORDANCE aims at realizing the concept of introducing OFDMA-based layer-1 technology and layer-2 protocols to provide a variety of desirable characteristics such as increased aggregate bandwidth and scalability, enhanced resource allocation flexibility, longer reach, lower equipment cost/complexity, and lower power consumption, while also supporting multiwavelength operation. In addition, it enables the convergence of the optical fiber infrastructure with standard wireless solutions, thus offering a way to integrate dominant wired and wireless technologies in a hybrid access network supporting seamless ubiquitous broadband services. More specifically, the OLT manages the assignment of traffic to a large number of subcarriers, which travel all along the optical distribution network to be demultiplexed only at the user side and vice versa. The subcarriers are grouped to form OFDM channels (with several tens or hundreds of subcarriers contained within one channel), each of them used for carrying traffic possibly by different providers and employing different optical, wireless, and copper-based technologies. ACCORDANCE was implemented using optical I/Q (oIQ) modulation at the OLT transmitter and simple direct detection at the ONU receiver for downstream communications and seeded intensity modulation (IM) at the ONU transmitter and coherent detection at the OLT receiver for upstream communications. Since all ONUs share the same (seeded) optical source, upstream OFDM signals are perfectly aligned and upstream OFDMA is made feasible.

3.2. Network Planning and Reconfiguration. To maximize the performance of FiWi networks and minimize their deployment costs, network planning and reconfiguration play a key role in achieving these design objectives. In this section, we describe a number of algorithms that help solve important FiWi network planning problems related to the optimal placement of ONUs, mitigation of the detrimental impact of wireless interferences for peer-to-peer communications between wireless end-users, and architectural modifications for the support of direct inter-ONU communications. Furthermore, we discuss the previously proposed reconfigurable FiWi network architectures that are able to respond to varying traffic loads.

3.2.1. ONU Placement. The optimal placement of ONUs is an important design objective of FiWi networks due to the fact that the cost of laying optical fiber is significantly higher than that of devices attached to either end of the optical fiber, for example, OLT.

Several heuristics to solve the problem of optimally placing ONUs in a FiWi access network consisting of a PON in tandem with a WiFi- or WiMAX-based WMN were studied in [39]. The first proposed heuristic is a greedy algorithm that aims at finding a suitable placement of multiple ONUs to minimize the average Euclidean distance between wireless endusers and their closest ONU; that is, this heuristic targets only the wireless front-end and does not take the fiber layout of the optical backhaul into account. The greedy algorithm starts with a given distribution of wireless end-users, which might be randomly or deterministically chosen, and consists of two phases. In the first phase, the algorithm identifies the primary ONU (i.e., closest $\mathrm{ONU}$ ) for each wireless end-user. In the second phase of the algorithm, for each primary ONU a set of wireless end-users is obtained such that the distances between the ONU and its wireless end-users are minimized. The performance of the proposed greedy algorithm was evaluated in the Wildhorse neighborhood of North Davis, California, under the assumption of random and deterministic ONU placement. The presented results show that the greedy algorithm performs better for deterministic rather than random ONU placement. The second proposed heuristic optimizes the placement of ONUs by means of simulated annealing, a widely used combinatorial optimization technique. For a given initial ONU distribution, this heuristic perturbs one of the high-cost ONUs to achieve a cost improvement in terms of average Euclidean distance between ONUs and wireless end-users. For each successful perturbation, wireless 


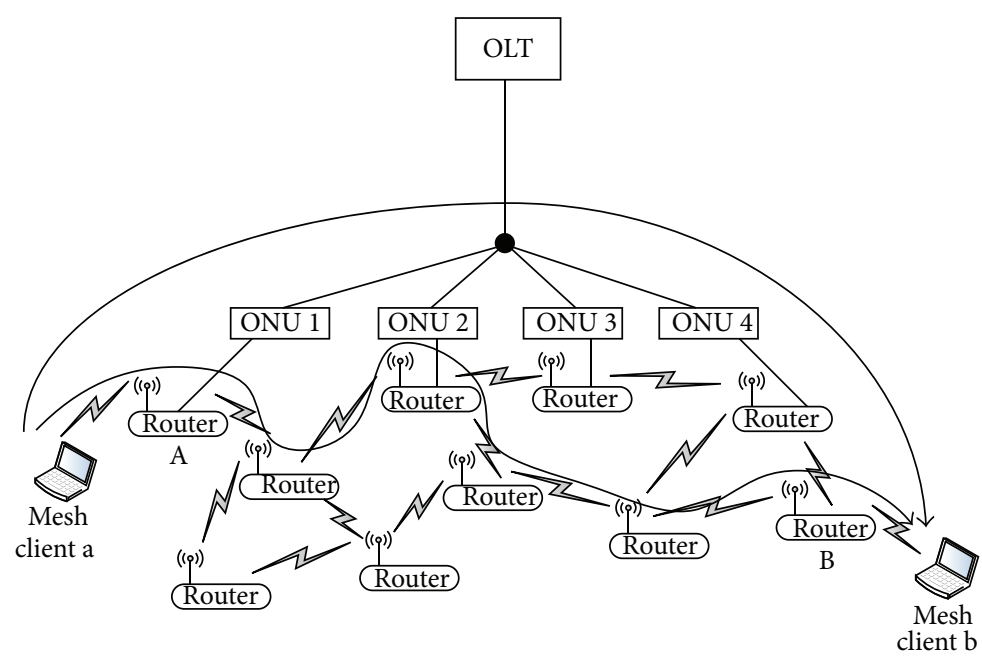

FIGURE 4: Peer-to-peer communications in FiWi access networks [42].

end-users are reassigned to their nearest ONU. The reported results show that simulated annealing is able to achieve an overall cost improvement of typically close to 5\% compared to the aforementioned greedy algorithm. Unlike the two previous algorithms, the third proposed heuristic also considers the cost of the fiber layout from ONUs to the OLT as well as interference in the wireless front-end. Toward this end, the proposed heuristic deploys the wireless access points (or base stations) uniformly, determines their transmission radius, and assigns channels to them such that the carrier-tointerference ratio does not drop below a certain threshold. After deploying the access points, the heuristic determines the number of ONUs needed to support the access points and deploys the ONUs according to the above-described greedy algorithm. Finally, the heuristic finds a minimum-cost spanning tree to lay fiber from the OLT to all ONUs.

A powerful mixed integer programming (MIP) model for the optimum placement of ONUs and WiMAX BSs in a PON/WiMAX-based FiWi network was developed in $[40,41]$. The presented MIP model minimizes the number of deployed ONUs and BSs by saving costs and reducing the interference among multiple BSs and nearby wireless users. Specifically, the objective is to minimize the total installation cost of all ONUs and BSs required, including cost of connecting the BSs to an ONU by means of optical fiber. Furthermore, the MIP problem takes into account a wide range of important constraints with regard to user assignment, ONU installation, BS installation, capacity, and channel assignment, as well as signal quality and interference. More precisely, each wireless user is associated with at most one BS and the distance between a given wireless user and its associated $\mathrm{BS}$ must be within the transmission range of that BS. Moreover, the number of channels assigned to each BS is large enough to serve its wireless users, while not exceeding the upper bound of channels assigned to any BS. Each BS must be connected to only one ONU and the capacity of each ONU is large enough to serve all traffic generated by its attached BS. Finally, the quality of the wireless signal has to be at least the threshold of an acceptable carrier-to-interference ratio. For tractability, the authors used Lagrangian relaxation to relax some of the constraints and solve the MIP problem with acceptable accuracy. The presented simulation results indicate that the ONU/BS placement problem is quite sensitive to a number of parameters, for example, number of available wireless channels and wireless user coverage ratio.

\subsubsection{Inter-ONU Communications}

Peer-to-Peer Communications. In FiWi networks, traffic may go from wireless end-users to the Internet or from one wireless client to another wireless client. In the former case, the throughput of FiWi networks is limited by the bandwidth bottleneck and interferences of communications in the wireless subnetwork. In the latter case, the bimodal nature of FiWi networks provides an opportunity to mitigate the detrimental impact of wireless interferences on the network throughput for peer-to-peer communications between two wireless endusers. In FiWi networks, peer-to-peer traffic can be carried in two ways: (i) either through the wireless front-end only or (ii) along a wireless-optical-wireless path [42]. As shown in Figure 4, suppose that wireless mesh client $a$ wants to communicate with mesh client $b$. The resultant peer-to-peer traffic can be routed either through the multihop wireless path within the wireless subnetwork or through the wirelessoptical-wireless path, whereby traffic is first sent from mesh client $a$ via its associated mesh router $A$ to its closest ONU 1 and is then forwarded upstream to the OLT. Subsequently, the OLT broadcasts the traffic downstream to all ONUs. Each receiving ONU determines to discard or forward the traffic according to the location of the destination mesh client. In our example, ONU 4 is closest to destination mesh client $b$ and thus ONU 4 will forward the arriving traffic to $b$ via its associated mesh router $B$, while all remaining ONUs discard the traffic coming from the OLT. Note that traffic forwarded along the wireless-optical-wireless path alleviates interferences in the wireless front-end, thereby allowing more 
traffic to be carried in the wireless segment and resulting in an increased network throughput. Also note that the capacity of the PON is much higher than that of the wireless front-end such that peer-to-peer traffic can be easily carried in the optical backhaul without suffering from any serious throughput penalty.

Clearly, to exploit the benefits of steering peer-to-peer traffic along wireless-optical-wireless paths, the placement of ONUs has a great impact on the achievable throughput of FiWi networks. In [42], the ONU placement was optimized with the objective to maximize the throughput of FiWi networks for peer-to-peer communications, assuming that single-radio single-channel wireless routers are deployed and ONUs can communicate with each other by sending traffic to the OLT, which in turn broadcasts traffic back to all ONUs. Given the location of the wireless mesh routers, the optimal placement of $K$ ONUs was found with the objective to minimize the total number of required wireless hops. Once the location of the $K$ ONUs is fixed, each wireless node calculates the shortest path (in terms of hops) within the wireless mesh front-end to each ONU and selects the ONU with the minimal hop count. To solve this optimization problem, the authors applied a tabu search based heuristic, a widely used metaheuristic algorithm for solving combinatorial problems. The presented simulation results show that under different network sizes the proposed tabu search heuristic outperforms random and fixed ONU placement schemes in terms of achievable network throughput, especially for an increasing number of ONUs.

FiWi versus WMN Networks. To measure the network throughput gain in FiWi networks, a linear programming based routing algorithm was proposed in [43]. The objective of the routing algorithm is to route traffic in the FiWi network such that network throughput is maximized. The proposed optimal routing algorithm yields a bound on the throughput gain in FiWi networks. Extensive simulations were conducted to study the network throughput gain in FiWi networks under peer-to-peer traffic among wireless mesh clients and compare the achievable throughput gain to conventional WMNs without any optical backhaul. The presented simulation results show that the network throughput gain of FiWi networks is zero compared with traditional WMNs when all traffic is destined to the Internet, that is, no peer-to-peer traffic, since the interference in the wireless front-end represents the major bandwidth bottleneck. In other words, when all the traffic goes to the Internet, the throughput of FiWi networks is the same as that of traditional WMNs. However, with increasing peer-to-peer traffic the interferences in the wireless mesh front-end increase and the throughput of WMNs decreases dramatically. In contrast, the throughput of FiWi networks decreases to a much lesser extent for increasing peer-to-peer traffic due to the above-described wireless-optical-wireless communications mode of FiWi networks. Thus, these findings show that FiWi networks are particularly beneficial for supporting peer-to-peer communications among wireless mesh clients and are able to achieve a significantly higher network throughput than conventional WMNs.
Direct Inter-ONU Communications. The throughput-delay performance of FiWi networks for peer-to-peer communications can be further improved by means of direct interONU communications. In conventional PONs, inter-ONU communications are done via the OLT. In [44], a new WDM/TDM PON-based FiWi network architecture was proposed and investigated to enable direct inter-ONU communications without going through the OLT. The proposed WDM/TDM PON architecture requires major hardware extensions at the OLT, ONUs, and remote node. More specifically, the OLT is equipped with an array of fixed-tuned transmitters and an array of fixed-tuned receivers, each operating on a different dedicated wavelength channel, for transmitting and receiving data to and from the ONUs. Each ONU deploys a pair of fixed-tuned transmitter and fixed-tuned receiver at dedicated upstream and downstream wavelength channels. In addition, each ONU uses a tunable transmitter and a fixedtuned receiver for inter-ONU communications. The interONU traffic from all ONUs is combined into one fiber by means of a passive combiner, which is attached to one of two input ports of an arrayed-waveguide grating (AWG). Both the combiner and AWG are located at the remote node of the WDM/TDM PON. The second input port of the AWG is used to interconnect the OLT with the ONUs for communications between the OLT and ONUs. The additional transceiver enables ONUs to send data directly to each other across the AWG rather than going through the OLT, resulting in an improved throughput-delay performance under peerto-peer traffic. At the downside, it must be mentioned that the proposed architecture is quite complex and costly. Not only do the required tunable transmitters add to the complexity and cost of each ONU but also might optical amplifiers become necessary to compensate for the coupling and insertion losses of the inserted combiner and AWG, thereby violating the unpowered nature of PONs and costsensitivity of access networks in general.

3.2.3. Reconfiguration. Previously, we have introduced an optical unidirectional WDM ring interconnecting multiple PONs integrated with a WiFi-based WMN. To adapt the considered FiWi network to traffic changes in different districts, reconfigurability was implemented in the optical backhaul $[27,45]$. Instead of overprovisioning based on the peak traffic demand, it is desirable to reallocate bandwidth among multiple PONs by utilizing tunable transceivers at the ONUs. For instance, let us assume that two PONs share the same optical tree subnetwork and one PON is highly loaded while the other PON is not, then by tuning the transmitting and receiving wavelengths of a highly loaded ONU associated with the former PON the ONU is enabled to join the latter lightly loaded PON in order to achieve load balancing between the two PONs. Toward this end, in the central hub of the FiWi network a network terminal continually monitors the buffer depth of each OLT for the downstream traffic. As a PON becomes heavily loaded, as indicated by the corresponding OLT buffer depth, the heavily loaded PON is instructed to deregister some ONUs and reregister them to the lightly loaded PON(s). Before an ONU is deregistered, its packets queued at the OLT will first be 
emptied. After an ONU is deregistered, the incoming traffic to that ONU is temporarily stored at the network terminal until the reregistration is achieved. To reduce the overhead of slow transceiver tuning times during reconfiguration and increase network throughput, the buffer size may be increased and a higher threshold for the reconfiguration trigger may be set, at the expense of longer queueing delays in the buffer.

3.3. Routing and QoS Continuity. The wireless mesh frontend of FiWi access networks provides multiple paths to route traffic coming from and going to the optical backhaul. In this section, we review a variety of proposed routing algorithms that aim at optimizing the network performance in terms of delay, throughput, packet loss, load balancing, and other important metrics such as path availability and power consumption. The considered routing algorithms cover either only the wireless front-end or both the wireless and optical domains of FiWi access networks. Furthermore, we elaborate on various techniques to provide service differentiation and end-to-end guaranteed QoS and enable QoS continuity across the optical-wireless interface of FiWi broadband access networks.

3.3.1. Wireless Routing Algorithms. In the following, we describe various proposed routing algorithms for the wireless front-end of FiWi access networks. All of the discussed wireless routing algorithms aim at finding the optimal path through a wireless mesh front-end by meeting one or more objectives.

$D A R A$. A proactive routing algorithm, referred to as delayaware routing algorithm (DARA), that minimizes the average packet delay between a router and any wireless mesh gateway was presented in $[39,40]$. DARA is a link-state routing algorithm, where each wireless mesh router and gateway periodically advertises its link conditions (i.e., traffic intensity and link capacity with time stamp) in link state advertisement (LSA) messages. Upon reception of an LSA message, each router/gateway predicts the traffic intensity, which is used to predict the state of the corresponding link until the next LSA message arrives. To make sure that the resultant link state prediction (LSP) is accurate, the authors proposed to use weighted moving average (WMA) to estimate the traffic intensity of a given link between the current LSA message and the next LSA message. Based on the LSP information, each wireless link is assigned a weight according to its predicted transmission, synchronization, and queuing delay. More precisely, links with higher predicted delays are given higher weights and vice versa. Subsequently, for each pair of router and gateway a single (or alternatively $K>1$ for load balancing) minimum-weight path(s) is (are) computed. In addition, DARA performs admission control, where a packet is admitted into the wireless mesh only if the predicted delay along the computed path is below a predetermined threshold. Otherwise, the packet is rejected.

The presented simulation results indicate that DARA outperforms other routing algorithms such as minimum hop routing, shortest path routing, and predictive throughput routing in terms of delay. Furthermore, DARA is able to improve load balancing and alleviate congestion compared to minimum hop and shortest path routing algorithms. It also improves the average hop count compared to the predictive throughput routing algorithm.

DDRA. The aforementioned DARA routing algorithm does not allow for any sort of QoS differentiation. To do so, the so-called delay-differentiated routing algorithm (DDRA) was introduced in [46]. The objective of DDRA is to decrease the delay of upstream transmissions to a server located at the OLT of a PON. Toward this end, the authors define the delay-toserver (DTS) as the time from a wireless mesh network client to the server. Traffic is divided into DTS-sensitive and DTSinsensitive traffic. Given that a significant proportion of the path delay generally occurs at the wireless bottleneck links between gateways and their adjacent wireless mesh routers, the delay performance can be improved by attaching external buffers to these routers. The external buffers can store DTSinsensitive data packets when the links are congested and forward them to the server when the links to the gateways become less congested. In doing so, under high traffic loads DDRA decreases the queuing delay of DTS-sensitive traffic by temporarily storing DTS-insensitive data packets in external buffers. A given ingress wireless router computes a path to a gateway, if the traffic is DTS-sensitive. In the case of DTSinsensitive traffic, a path from the ingress router to one of the external buffers is computed. Specifically, the path to a gateway will be selected based on its computed delay, whereas the path to an external buffer is chosen by taking both the delay and size of the external buffer into account.

The performance of DDRA and DARA was compared by means of simulation. The results reported in [46] show that under light loads DDRA and DARA achieve roughly the same delay performance for DTS-sensitive traffic, though DTSinsensitive traffic suffers from a slightly higher delay. Under increasing traffic loads, DDRA outperforms DARA in terms of delay and can support nearly 1.2 times the maximum load that DARA can handle, at the expense of additional hardware requirements at some of the wireless mesh routers.

CaDAR. The capacity of wireless mesh networks is limited. This is particularly true for single-radio wireless mesh networks, where each mesh node is equipped with only one radio, whose capacity needs to be distributed among all the node's outgoing links by means of time division multiple access (TDMA). By assigning time slots of different duration to a node's outgoing links according to given traffic demands, each of these links has a different capacity. In [47, 48], a new routing algorithm, called capacity and delay aware routing $(C a D A R)$, was proposed with the objective to distribute the radio capacity of a single-radio wireless mesh network node optimally among its outgoing links such that the average delay is minimized. Specifically, in CaDAR, each node informs its neighboring nodes about the current traffic load of all its outgoing links via periodic LSA messages. Based on the advertised link loads, the CaDAR algorithm determines the fraction of a node's radio capacity that should be assigned to each of its outgoing links. Subsequently, each link is assigned its delay as weight and CaDAR computes the shortest delay 
path for each pair of wireless mesh router and gateway. Similar to DARA, CaDAR may deploy admission control and WMA to estimate the traffic load of each link. It was shown for a 25-node single-radio IEEE 802.11g WLAN based mesh network that CaDAR can support nearly three times the maximum load DARA can handle while maintaining low delays.

To increase the capacity of the wireless front-end and better match it to the optical backhaul, one promising approach is to deploy multiple radios at nodes that carry higher traffic volumes. In [49], the problem of optimum placement of a second radio at selected wireless routers/gateways was formulated as an integer linear program (ILP) with the objective to maximize the throughput-delay ratio of the wireless frontend. By means of simulation it was shown that the maximum throughput performance of CaDAR can be almost doubled if only the gateways deploy an additional second radio while leaving all mesh routers with a single radio. Equipping some of the wireless mesh routers with an additional radio does not lead to an increased maximum throughput due to the fact that the bottleneck links remain the same; however, it helps reduce the average delay of the network to some extent. In fact, the throughput-delay ratio of the wireless frontend increases for a growing number of multiple-radio mesh routers.

In a wireless front-end with multiple-radio mesh nodes, it is important to assign the wireless channels efficiently such that contention is reduced. A channel assignment scheme that balances traffic loads among wireless channels to minimize contention was investigated in [50]. The proposed channel assignment scheme performs load balancing of traffic flows across different channels in order to maintain a similar level of contention on each channel. Using CaDAR for routing, it was shown by means of simulation that the proposed flowaware channel assignment scheme effectively balances the load across different wireless channels, resulting in a reduced contention throughout the wireless front-end.

RADAR. In FiWi access networks, packet loss may occur due to various failures, for example, gateway or OLT failure. To cope with packet loss, the so-called risk-and-delay aware routing (RADAR) algorithm was studied in [40]. RADAR is an extension of the above-mentioned DARA routing algorithm. Similar to DARA, each wireless router/gateway advertises the wireless link states in periodic LSA messages and assigns weights to wireless links according to their delays for minimum delay path computation. Unlike DARA, each mesh router maintains a risk list to keep track of failures. If a failure occurs, the risk list is updated and subsequent packets are rerouted. Specifically, a risk list contains the following six fields:

(i) path number,

(ii) primary gateway group (PGG),

(iii) secondary gateway group (SGG),

(iv) tertiary gateway group (TGG), (v) path status,

(vi) path delay,

whereby the path status can be either live or stale. The primary gateway for a given mesh router is the gateway with the minimum delay path. The PGG contains paths to the primary gateway and other gateways connected to the same ONU of a back-end PON. The SGG contains paths to gateways that are connected to different ONUs but the same OLT as the PGG. The TGG contains paths to gateways that are connected to a different OLT.

In the event of one or more FiWi network failures, the risk list of affected mesh routers is updated accordingly. More precisely, if the primary gateway fails, then all the paths to that gateway become stale and packets destined for the primary gateway are rerouted through live PGG paths. If the ONU of the PGG paths fails, all PGG paths become stale and packets are rerouted along live SGG paths. If the common OLT of the SGG paths fails, all SGG paths become stale and packets are rerouted along live TGG paths.

RADAR provides protection against multiple wireless front-end and/or optical back-end network failures. It minimizes the average packet delay in the wireless front-end and reduces the packet loss of FiWi access networks significantly. It was shown by means of simulation that in terms of both average delay and packet loss RADAR clearly outperforms minimum hop, shortest path, and predictive throughput routing algorithms for a wide range of failure scenarios such as gateway, ONU, and OLT failures.

3.3.2. Integrated Routing Algorithms. Integrated routing algorithms compute paths across the optical-wireless interface by taking both the wireless and optical domains into account.

CaDAR with Optical Delay Awareness. The CaDAR routing algorithm, originally intended only for the wireless frontend as explained above, can be extended to perform integrated path computation across both the wireless and optical domains of FiWi access networks. In [51], CaDAR was used in conjunction with interleaved polling with adaptive cycle time (IPACT) with constant credit service as DBA algorithm in a backhaul EPON. IPACT is used to arbitrate the communication between OLT and ONUs. For downstream data transmissions to a wireless destination node, the OLT selects a gateway such that data packets travel on the minimum delay path across the EPON and wireless mesh frontend. To select the correct gateway, all gateways periodically send LSA messages to the OLT and the OLT computes the minimum delay path across the EPON and wireless mesh front-end. To calculate the minimum delay path for upstream data transmissions from wireless mesh clients to the OLT, a gateway takes the current load of its collocated ONU into account and estimates the upstream delay on the EPON. Subsequently, the gateway disseminates the estimated delay throughout the wireless mesh front-end via periodic LSA messages. A given wireless mesh source node uses this information for computation of the minimum delay path to the OLT. 
The presented simulation results investigated the average packet delay for CaDAR under the assumption of equally and unequally loaded ONUs. It was shown that when the load of a particular ONU is increased, more traffic is diverted toward gateways collocated with other ONUs. When all ONUs have equal load, traffic from wireless mesh source nodes tends to go toward the closest gateway using fewer wireless hops, which translates into shorter delays.

Availability-Aware Routing. Apart from capacity, one of the major differences between the optical and wireless parts of FiWi access networks is the fact that optical wired links are much more reliable than their wireless counterparts, whose transmission characteristics depend on a number of different parameters. This major difference between optical fiber and wireless links should also be taken into account in the design of integrated routing algorithms for FiWi access networks.

An availability-aware routing algorithm for PON/wireless mesh based FiWi networks was examined in [52]. Availability is the probability that a connection will be found in the operating state at a random time in the future. It is given by

$$
\text { Availability }=\frac{\text { MTTF }}{\text { MTTF }+ \text { MTTR }}
$$

where MTTF denotes the mean time to failure and MTTR denotes the mean time to repair. Availability is in general affected by many factors and is an important parameter in the service level agreement (SLA) between network operators and customers. The availability of a path can be calculated as the product of the availabilities of the individual links, whereby the most available path is defined as the path with the highest availability from the source node to the destination node of a given connection request. The performance of the availability-aware routing algorithm was investigated for different link availabilities and compared to the shortest path routing under uniform traffic. It was shown by means of simulation that the availability-aware routing algorithm is able to achieve a higher average availability for the selected paths than the shortest path routing, especially at low traffic loads. However, availability-aware routing is less bandwidthefficient than the shortest path routing due to an increased mean number of hops.

A link availability model for FiWi access networks was developed in [53] and its performance was analyzed for various routing algorithms, including DARA, CaDAR, availability-aware routing, and multipath routing. The obtained results show that shortest path routing using link unavailability as the link cost metric significantly improves the availability of the selected paths. Moreover, it was shown that multipath routing helps further improve the availability compared to single-path routing.

Multipath Routing. Multipath routing and its implications have been studied in greater detail in [54]. With multipath routing, data packets can be sent upstream to the OLT along multiple paths in the wireless front-end, resulting in improved load balancing and fault tolerance as well as higher network throughput. However, these data packets may arrive at the OLT out of order due to the fact that each path in general has a different delay. As a consequence, the out-of-order packets deteriorate the performance of the transmission control protocol (TCP). In PON-based FiWi access networks, the OLT serves as a convergence node of all upstream data flows and may as such resequence arriving packets to some extent, thereby mitigating the detrimental impact of out-of-order packet delivery and increasing TCP throughput.

In [54], the authors proposed an optimal flow assignment and fast packet resequencing algorithm in order to reduce the out-of-order probability of upstream packets injected by the OLT to the Internet. In this approach, packets belonging to the same flow are sent to the OLT along multiple paths. The delay along different paths may be different and may vary over time. The OLT maintains two first-in-first-out (FIFO) priority queues with preemptive priority scheduling. Out-oforder packets are stored in the high-priority queue, while the remaining packets are put in the low-priority queue. Flows are optimally assigned among the different paths with the objective to minimize the out-of-order probability beyond the OLT subject to given packet delay requirements.

3.3.3. Energy-Aware Routing. FiWi networks and access networks in general suffer from a major shortcoming. In today's Internet, the total energy consumption is dominated by access networks and as access rates of tens of $\mathrm{Mb} / \mathrm{s}$ become commonplace, it will be necessary to improve their energy efficiency in order to avoid a significantly increased greenhouse footprint of the Internet [55]. "Green" energyefficient FiWi access networks can be realized by putting selected nodes, for example, ONUs and wireless mesh routers, to a low-power (sleep) state in a coordinated manner during low traffic load hours in order to save energy [56].

A couple of interesting techniques to design green EPON/WLAN-mesh based FiWi access networks were studied in $[57,58]$. The authors proposed a centralized sleeping mechanism that allows the OLT to put low-load ONUs asleep. Specifically, the OLT maintains two watermarks for the traffic load at each ONU, one low watermark and one high watermark. The OLT observes the traffic load at each ONU by monitoring the length of the corresponding queues. If a given ONU operates under the low watermark, the OLT puts it asleep and the wireless front-end is used to reroute traffic to other active ONUs. The OLT wakes a sleeping ONU up when the traffic load of the active ONUs crosses the high watermark in order to cope with the increased traffic load. The authors formulated this problem as a mixed integer linear program (MILP) to determine the minimum number of ONUs that need to be kept active to support a given traffic load, while all remaining ONUs are put in the sleep mode, thereby minimizing the power consumption of the FiWi access network.

The model can be solved for small FiWi network instances. However, for FiWi access networks with a larger number of nodes and higher traffic loads, heuristics are needed to solve the problem. Toward this end, the authors investigated an energy-aware routing algorithm. The objective of the proposed energy-aware routing algorithm is to 
use already existent paths and put the other FiWi network segments asleep. To do so, the residual capacity of each link is assigned as its weight and LSA messages are periodically sent to inform all other network nodes about the current residual capacity of each link. Based on this information, data packets are routed along the lowest residual capacity path between each pair of source and destination nodes. Note, however, that this approach increases the average path length and path delay since the algorithm in general provides routes that are longer than the corresponding shortest paths. To mitigate this problem, the authors proposed to use a so-called hop offset. Given a path of $n$ hops, for each hop a hop offset $m \geq 1$ is added to the path cost; that is, $n \times m$ is added to the original cost of the path. The presented simulation results indicate that a significant number of ONUs of an EPON/WLANbased mesh FiWi access network can be put asleep without compromising its delay performance.

3.3.4. QoS Continuity. A plethora of enabling optical and wireless network technologies and QoS provisioning techniques exists for FiWi access networks. In this section, we highlight some of the recently proposed techniques to provide QoS continuity across the optical-wireless interface of FiWi access networks. For a more detailed and comprehensive description of QoS provisioning techniques for FiWi access networks the interested reader is referred to [59].

QoS-Aware Scheduling. A centralized QoS-aware scheduling algorithm was proposed in [60] to deliver services to both wireline and wireless users of an integrated EPON-WiMAX network. For QoS provisioning, the proposed scheduling algorithm utilizes the integrated ONU/WiMAX outdoor unit (ODU) to collect the resource information and service requests from all wireless subscribers. Whenever the wireless link conditions change, a WiMAX subscriber station sends a bandwidth request message to its associated ODU to update its service requirements. The collocated ONU aggregates the received bandwidth requests from multiple subscribers into a small number of multipoint control protocol (MPCP) REPORT messages and sends them upstream to the hybrid OLT/WiMAX base station, which acts as the central controller of the integrated EPON/WiMAX network. The aggregation of multiple bandwidth request messages into a small number of REPORT messages helps reduce the signaling overhead and achieve an improved throughput-delay performance. For each polling cycle, the central controller schedules the received bandwidth requests based on their traffic class and informs the WiMAX subscriber stations about the resultant upstream transmission schedule.

The presented simulation results show that the centralized QoS-aware scheduling algorithm is able to improve both network delay and throughput by roughly $10 \%$ compared to a distributed multihop scheduling algorithm, where each hop makes its own local transmission decision without any global knowledge of the status of all wireless links.

Enhanced MPCP and Admission Control. A resource management framework with the objective of maximizing the resource utilization and optimizing the QoS performance in both the optical and wireless segments of an integrated EPON/WiMAX network was investigated in [61]. The framework comprises two parts. The first part is the so-called enhanced MPCP (E-MPCP), which aims at improving the signaling between EPON and WiMAX. The second part is called the integrated optical wireless admission control (IOW$A C)$ scheme, which is used to provide integrated bandwidth allocation in an EPON/WiMAX network.

In this approach, the collocated EPON ONU and WiMAX base station are integrated into a single device referred to as access gateway. In E-MPCP, the GATE message is modified such that it contains an additional 3-byte field called the next cycle time, which denotes the time interval between two consecutive polling operations of a given access gateway. In doing so, the access gateway knows the estimated waiting time for its next poll and can thus calculate the transmission delay in the optical domain more accurately. The IOW-AC works as follows. Upon reception of a new service request, the access gateway calculates the transmission and propagation delays by taking the wireless network conditions and traffic profile into account. Subsequently, the access gateway calculates the queuing delay based on the current buffer occupancy. Finally, the access gateway also takes the aforementioned transmission delay in the optical segment into account and determines the estimated overall delay across both WiMAX and EPON networks. If the overall delay is smaller than a prespecified delay bound the service request is accepted. Otherwise, the service request is rejected.

Simulation results were presented to compare the proposed integrated resource management framework with a conventional resource management scheme that controls the EPON and WiMAX networks separately from each other. The reported results show that E-MPCP and IOW-AC are superior to the separated resource management scheme in terms of channel utilization and dropping probability for both best-effort and real-time traffic. However, it is important to note that the performance of the integrated resource management framework largely depends on the network size and traffic profile.

QoS Mapping. One important aspect of QoS continuity across the optical-wireless interface of FiWi access networks is the appropriate mapping of traffic classes of different optical and wireless broadband access technologies. In general, optical and wireless broadband technologies differ in the number and/or type of traffic classes. For instance, EPON deploys the eight priority traffic classes defined in IEEE standard 802.1D. On the other hand, fixed and mobile WiMAX defines four and five scheduling services to support different traffic classes, respectively, while in IEEE 802.11e QoS-enabled WLANs four different access categories are used, each associated with a different wireless channel access priority. These different priority queues, scheduling services, and access categories are used to meet the specific QoS requirements of different services and applications such as voice, video, email, web browsing, and file transfer. To provide end-to-end QoS support in FiWi access networks consisting of cascaded EPON, WiMAX, and/or WLAN networks for a variety of 
services and applications with different QoS requirements, the priority queues, scheduling services, and access categories must be mapped properly, which can be easily done in a straightforward manner [62].

Optical Burst Wireless Mesh Architecture. A metropolitan area FiWi network architecture called optical burst wireless mesh architecture (OBWMA), which interconnects multiple WMNs at the user access side through an optical burst switching (OBS) core network, was proposed in [63-65]. One or more gateways of each WMN are connected to an OBS edge node. To provide end-to-end guaranteed QoS, OBWMA accepts a new flow request only when the flow end-to-end delay and bandwidth requirements can be met in the WMN. In the WMN, each mesh node has two radios with several orthogonal channels per radio. The wireless channels are statically assigned to each radio such that interference is minimized. The OBS core network is a WDM ring network consisting of several OBS edge nodes. Each OBS edge node aggregates received data packets into bursts based on the packets' destinations.

OBWMA supports two traffic classes. The high-priority traffic class has QoS requirements with respect to delay and bandwidth, while the low-priority traffic class does not have any QoS requirements (i.e., best-effort traffic). In the $\mathrm{WMN}$, a certain percentage of the link capacity is allocated to each traffic class at every mesh node and either traffic class is allowed to exceed its allocated bandwidth only if the other traffic class has not reached its maximum bandwidth. Otherwise, excess traffic needs to be dropped. To enable lossfree transport of high-priority traffic across the OBS core network, each OBS network node is assigned a number of local wavelength channels that are dedicated to the transmission of high-priority traffic. Low-priority traffic is sent on any of the remaining contention-based wavelength channels or on the aforementioned local wavelength channels, provided that there is no high-priority traffic. To ensure end-to-end guaranteed QoS across the WMN and OBS networks, a control bridge is deployed at the OBS edge node, which has global knowledge about the OBWMA network resources. The control bridge performs QoS mapping between the WMN and OBS networks of OBWMA.

The performance of the OBWMA network was investigated in terms of throughput, delay, and packet loss by means of simulation. The obtained results show that at low traffic loads the end-to-end delay of the OBWMA network is dominated by the OBS core network. This is due to the fact that the burst assembly takes more time at low traffic loads. Conversely, at high traffic loads the WMN gets increasingly congested, resulting in an increased end-to-end delay. Furthermore, it was shown that the above-described bandwidth allocation schemes help decrease the packet loss and increase the per-flow throughput of the network.

\section{Recent Progress}

The design of energy-efficient "green" FiWi access networks has been receiving considerable attention [66]. An overview of recently proposed energy-efficient architectures as well as energy-efficient MAC and routing protocols for FiWi access networks was provided in [67]. The authors also proposed an OBS based dynamic bandwidth allocation (DBA) protocol for the energy-efficient integration of long-reach EPON and long term evolution-advanced (LTE-A) technologies and investigated the tradeoffs between energy savings and QoS guarantees. The simulation and experimental work in [68] has shed some light on the tradeoff between energy saving and QoS support in FiWi access networks. A unified analytical framework for the throughput-delay performance evaluation of a wide range of FiWi network routing algorithms was developed in $[69,70]$, taking emerging IEEE 802.1lac VHT WLAN technologies and PON fiber faults into account.

Beside the energy-efficient design of FiWi access networks, their survivability has been another recent important research area. A variety of advanced survivability techniques for PONs and FiWi access networks were investigated in $[71,72]$. A centralized architecture incorporating RoF and cognitive radio technologies into a unified network called cognitive WLAN over fibers by replacing WLAN APs with RAUs was presented in [73], including a testbed architecture built on a PON and software defined radio (SDR) platform. Furthermore, next-generation PONs (NG-PONs) are expected to play an important role in the support of coordinated multipoint (CoMP) transmission and reception in $4 \mathrm{G} \mathrm{LTE}$ /WiMAX networks. It was shown in [74] that by using so-called XG-PONs, which offer symmetrical data rates of $10 \mathrm{~Gb} / \mathrm{s}$, instead of point-to-point fibers, fiber backhaul deployment costs in $4 \mathrm{G}$ CoMP architectures can be reduced by up to $80 \%$. Network coding based energy management solutions for NG-PONs were presented in [75]. The capacity and delay performance of various types of NG-PON were analyzed in [76]. A scheduling and DBA scheme that allows the coexistence of high-speed $10 \mathrm{~Gb} / \mathrm{s}$ EPON and multichannel WDM PON on the same optical infrastructure was studied in [77]. Different low-latency polling schemes for long-reach PONs were analyzed in $[78,79]$. A costeffective and fully centralized RoF network architecture based on integrated long-reach WDM NG-PON, with an optical fiber range of $100 \mathrm{~km}$, and LTE/WiMAX networks, where the optical and wireless transmission impairment compensation is done only at the $\mathrm{CO}$, was proposed and experimentally investigated for so-called quintuple-play services (high-speed data, voice, television, management, and home security) [80]. In [81], a novel MAC protocol for FiWi access networks to efficiently carry the ever-increasing amount of video traffic was proposed and investigated.

Recently, the integration of wireless and fiber optic sensors into FiWi access networks has begun to receive increasing attention. In [82], a hybrid approach combining genetic algorithm and tabu search techniques was proposed to determine the optimal placement of ONUs in hybrid PON-wireless sensor network (WSN) based networks, whereby the ONUs' high power and processing capabilities were used to serve as cluster heads with the objective to minimize the wireless sensors energy consumption. In [83], a DBA algorithm was developed for a hybrid PON-WSN network, where ONUs are connected to FTTH terminals or to WSN cluster heads for remote monitoring systems in a ubiquitous city, for 
example, medical sensors in a hospital monitoring system. The integration of solar-powered wireless sensors and lowpower fiber optic sensors into a PON was experimentally demonstrated in [84]. Finally, the research and development vision of an ideal access system architecture was outlined in [85], identifying the following three key design goals of future broadband access network architectures: (i) "adaptable," (ii) "dependable," and (iii) "ecoconscious." Toward this end, the authors concluded that passive (optical) network infrastructures should be used as much as possible and passive equipment may be shared in order to enable cost reduction, promote open and fair service competition, create novel value-added services, and introduce original business ideas for the realization of future-proof broadband access networks based on both wireless and fiber media.

\section{The Road Ahead}

In the PON community, researchers from both industry and academia have begun to contemplate on what the future may hold for evolutionary NG-PON1 and disruptive NG-PON2 technologies. Recently, at the 2013 OFC/NFOEC conference, a workshop on "Post NG-PON2: Is it More About Capacity or Something Else?" was held to find out whether it is reasonable to ever increase the system bandwidth or rather explore service and application as well as business and operation related aspects, which motivate access technology to move into a substantially different direction in the long run than continued capacity upgrades. Clearly, NG-PONs are expected to play an important role in the support of CoMP coordination schemes among base stations in 4G LTE/WiMAX networks. In fact, in emerging LTE-A heterogeneous networks (HetNets), where femtocells with small, inexpensive, and lowpower BSs are introduced to supplement existing macrocells for the sake of an improved (indoor) coverage, enhanced celledge user performance, and boosted spectral efficiency per area unit, a cellular paradigm shift is required that recognizes the importance of high-speed backhaul connections, given that most $4 \mathrm{G}$ research so far has been focusing on the achievable performance gains in the wireless front-end only without looking into the details of backhaul implementations and possible backhaul bottlenecks [86]. Very recently, however, in their seminal work on quantifying the impact of different backhaul topologies (mesh versus tree) and backhaul technologies (e.g., WDM PON) on the performance of cellular networks, Biermann et al. have shown that ultimately the major factor limiting CoMP performance in $4 \mathrm{G}$ mobile networks is latency rather than capacity of the backhaul [87].

To help identify open key research challenges for NGPONs and converged FiWi broadband access networks, it is important to consider emerging trends in related areas in order to shift the post NG-PON2 research focus from mere capacity provisioning to more lucrative solution offerings by developing holistic groundbreaking solutions across multiple economic sectors other than telecommunications per se. Toward this end, in the remainder of this paper we will first elaborate on emerging trends and open challenges for FiWi networking research. More interestingly, to better comprehend the true potential of the aforementioned emerging trends, it is helpful to put them into a wider nontechnical context and see how they fit into the bigger picture of present (and past) economic recessions. The current global crisis is far from unexpected but rather represents a recurrent historical event that is typical for capitalist economies. As recently explained by Perez in her excellent work on the implications of financial collapses [88], there have been four previous situations equivalent to the current crisis in the past two centuries since the first industrial revolution. Figure 5 illustrates the historical recurrence of recessions as turning points, where roughly every 50 years the installation period of new technologies, the time when financial capital shapes the economy, has led each time to a major bubble followed by a major crash. This is the time for states to come back actively to change the focus from the stock market indices to the job-creating expansion of the real economy and increase in social wellbeing and to take convergent and synergistic actions that will lead markets and society to the next golden age, or deployment period, where the benefits of new technologies are fully realized across the entire economy, thereby offering a vast innovation and growth potential across multiple economic sectors. Toward this end, according to Perez, it is vital to expand Internet access either with FTTH or with wireless broadband access and combine information and communications technologies (ICTs) with massive "green" innovations in order to usher in a sustainable global golden age, which appears to be the only sustainable means to both stimulate the US and European economies and allow the incorporation of hundreds of millions of new consumers of a rapidly rising global middle class with more sustainable lifestyles and consumption patterns than in the past. In this paper, we argue that FiWi access networks will represent a cornerstone of future broadband installations and explore ways of how they can be deployed across relevant economic sectors other than telecommunications per se. After elaborating on the rationale behind the Third Industrial Revolution and its underlying Energy Internet, we will describe in more detail how Internet technology and renewable energies are beginning to merge and help realize the vision of the smart grid and create new exciting opportunities for telecommunication service providers, municipalities, and utilities to roll out dependable low-latency FiWi based smart grid communications infrastructures and split the value chain into multitier business models.

5.1. Emerging Trends and Open Challenges. The explosion of mobile data traffic has mandated the need for a new cellular architecture. Femtocells, which need to be more autonomous and self-adaptive than traditional small cells, are now widely deployed as small, inexpensive, and low-power BSs. Beside economic and regulatory issues, the new key challenges arising in femtocell deployments are (i) interference coordination, (ii) cell association and biasing, (iii) mobility and soft handover, and (iv) self-organizing networks (SONs) [89]. Key to the cost-effective deployment and operation of small-cell networks will be the sharing of already existing high-capacity FTTx backhaul infrastructures [90]. The importance of highcapacity and low-latency fiber backhaul infrastructures in HetNets is also emphasized by the introduction of CoMP, one 


\begin{tabular}{|c|c|c|c|c|c|}
\hline & & Installation period & Turning poi & Deployment period & \\
\hline $\begin{array}{l}\text { Great } \\
\text { surge }\end{array}$ & $\begin{array}{l}\text { Date } \\
\text { technologies } \\
\text { core country }\end{array}$ & Bubble prosperity & Recessions & "Golden age" prosperity & \\
\hline 1 st & $\begin{array}{l}1771 \\
\text { The industrial } \\
\text { revolution } \\
\text { Britain }\end{array}$ & Canal mania & $\begin{array}{cc}1 \\
1793-97 \\
1 \\
1 & 1 \\
1 & 1 \\
1 & 1\end{array}$ & $\begin{array}{l}\text { Great } \\
\text { British leap }\end{array}$ & $\begin{aligned} 1 \\
1 \\
1 \\
1 \\
1 \\
1 \\
1\end{aligned}$ \\
\hline 2 nd & $\begin{array}{l}1829 \\
\text { Age of steam } \\
\text { and railways } \\
\text { Britain }\end{array}$ & Railway mania & 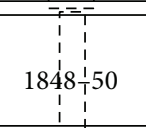 & The Victorian boom & $\begin{array}{cc}1 \\
1 \\
1 \\
1 \\
1 \\
1 \\
-1\end{array}$ \\
\hline $3 \mathrm{rd}$ & $\begin{array}{l}1875 \text { Age of steel and } \\
\text { heavy engineering } \\
\text { Britain/USA } \\
\text { Germany }\end{array}$ & $\begin{array}{l}\text { London funded global market } \\
\text { infrastructure build-up } \\
\text { (Argentina, Australia, and USA) }\end{array}$ & $\begin{array}{cc}1 \\
1 & 1 \\
1 & 1 \\
18190-95 \\
1 \\
1 \\
1 \\
\end{array}$ & $\begin{array}{l}\text { Belle Époque (Europe) } \\
\text { “progressive era” (USA) }\end{array}$ & $\begin{aligned}-1 \\
1 \\
1 \\
1 \\
1 \\
1 \\
1 \\
1\end{aligned}$ \\
\hline 4 th & $\begin{array}{l}1908 \\
\text { Age of oil, autos, } \\
\text { and mass } \\
\text { production/USA }\end{array}$ & $\begin{array}{r}\text { The roaring twenties in USA } \\
\text { autos, housing, radio } \\
\text { aviation, and electricity }\end{array}$ & $\begin{array}{c}\text { Europe } \\
1929-33 \\
\text { USA } \\
1929-43\end{array}$ & $\begin{array}{l}\text { I } \\
\text { Postwar } \\
\text { golden age } \\
\text { I } \\
\end{array}$ & $\begin{aligned}- \\
1 \\
1 \\
1 \\
1 \\
1 \\
-1\end{aligned}$ \\
\hline 5 th & $\begin{array}{l}1971 \\
\text { The ICT } \\
\text { revolution } \\
\text { USA }\end{array}$ & $\begin{array}{r}\text { Emerging markets } \\
\text { dotcom and Internet mania } \\
\text { real estate and financial casino }\end{array}$ & $\begin{array}{c}2000 \text { and } \\
2007-08 \text { ! } \\
\text { i }\end{array}$ & $\begin{array}{l}\text { Sustainable global } \\
\text { golden age? }\end{array}$ & $\begin{aligned}- \\
1 \\
1 \\
1 \\
1 \\
1 \\
1\end{aligned}$ \\
\hline
\end{tabular}

FIGURE 5: Historical recurrence of once-in-a-half-century bubble and golden age prosperities [88].

of the important performance-enhancing features of 3GPP LTE-A Release-11, which will have a major impact on the backhaul and will demand a careful design of backhaul links between BSs in order to provide high data rates and very low latency in the range of $1 \mathrm{~ms}$ or lower for the efficient coordination of transmission and reception among multiple BSs [91]. When migrating from macro-only to HetNet environments, mobility management becomes more challenging due to increased interference and decreased (femto)cell radius. According to [92], there is a clear trend toward including additional mobility enhancements in future LTE releases to ensure a smooth migration from traditional network controlled to user equipment (UE) assisted mobility as a remedy for reducing signaling overhead via fiber interconnections between macrocells and small cells. Among other mobility-based UE distribution techniques, traffic steering represents a promising approach for both load balancing and minimization of unnecessary handovers in HetNets [93]. Moreover, the introduction of SON technologies holds great promise to not only optimize network capacity, coverage, and service quality but also to substantially reduce (i) the complexity of interference coordination and, arguably more importantly, (ii) both operational and capital expenditures (OPEX/CAPEX) of LTE-A HetNets by minimizing human intervention [94]. Among the various SON functionalities, self-healing has so far received the least attention as opposed to widely studied self-configuration and self-optimization, making the development of real-time fault-compensation algorithms a key research topic in LTE-A HetNets [95].

Recall from above that the vast majority of previous FiWi network studies considered architectures based on low-cost simple EPON fiber backhaul and capacity-centric WLAN solutions. With the advent of high-speed IEEE 802.3av
10G-EPON and commercially available multichannel WDM PONs in conjunction with the emergence of Gigabit-class IEEE 802.11ac VHT WLAN technologies providing raw data rates of up to $6900 \mathrm{Mb} / \mathrm{s}$, future FiWi access networks will give rise to fiber backhaul and wireless front-end infrastructures of unprecedented capacity. In the past, $\mathrm{WiFi}$ and cellular radio access networks (RANs) have been in constant competition, until recently when tighter integration of both capacity-centric WLAN and coverage-centric mobile networks has emerged as a necessary paradigm [96]. The WLAN mesh front-end of FiWi access networks may be used to provide WiFi offloading of rapidly increasing mobile data traffic from cellular networks, thereby avoiding the need for capacity upgrades while at the same time helping reduce the complexity of future mobile networks. An interesting precursor of this trend is Deutsche Telekom's recently announced deal with crowdsourced WiFi hotspot provider FON, whose subscribers have signed up to mutually share their home broadband services in order to provide free "WiFi To Go" service on millions of hotspots worldwide (visit http://www.fon.com/ for further information). Complementing fast evolving HetNets with already widely deployed WiFi access points in addition to femtocells, which together are projected to carry over $60 \%$ of the global data traffic by 2015, represents a key aspect of the strategy of today's operators to offload mobile data traffic from their cellular networks [97]. It was recently shown by means of real-world iPhone measurements that significant offloading as well as battery power saving gains can be obtained if data transfers are delayed with some deadline until users enter a WiFi zone, though the achievable performance gains highly depend on given user mobility and spatial/temporal WiFi coverage patterns [98]. Beside traffic-aware scheduling, 
backhaul sharing is gaining significant importance to enhance the performance of cellular networks overlaid with $\mathrm{WiFi}$ offloading hotspots [96].

Another important trend is the convergence of mobile cellular networks and WSNs in order to support machineto-machine (M2M) communications. It was shown by means of simulation that the throughput-delay performance and lifetime of WSNs can be remarkably increased in such a converged network, but a number of essential issues still need to be resolved, most notably jointly optimized channel access techniques, two-level resource allocation schemes, and robust reselection algorithms [99]. Especially for eventdriven M2M communications, where a large number of devices become activated within a short period of time, traffic-aware random MAC protocols were shown to provide superior delay performance and robustness [100]. According to a recent OECD report on the future digital economy, one of the most promising applications of M2M communications is smart metering, which represents one of the first steps toward realizing the vision of the smart power grid [101]. The European Union (EU) has mandated the use of smart meters by 2020 in Directive 2006/32/EC, creating a market for around 180 million meters at a rate of one per household. Beside wireless technologies, the superior security and immunity features of PON based communications infrastructures, which are already installed in many countries, will be leveraged for the realization of large-scale sensor-actuator networks in support of future smart grid applications [102]. Today, Internet technology and renewable energies are beginning to merge in Europe, North America, and other regions worldwide in order to create an interactive, integrated, and seamless Energy Internet infrastructure for the so-called Third Industrial Revolution (TIR) economy, which goes well beyond current austerity measures and has been officially endorsed by the European Commission as economic growth roadmap toward a competitive low carbon society by 2050 [103], as discussed in more detail next.

\subsection{The Third Industrial Revolution (TIR), Energy Internet,} and Smart Grid. Many vital building blocks, organizations, and activities of today's society depend on the continued operation of various large and widespread critical infrastructures, including telecommunications networks and transportation systems. In particular, energy generation and distribution systems play a crucial role. Electrical power grids represent one of the most important critical infrastructures of our society. Current power grids with their aging infrastructure become increasingly unreliable and are poorly suited to face increasingly frequent outages, for example, the three-day blackout due to trees falling on power lines in the Washington D.C. area early July 2012, the lengthy power blackout in the states of New York and New Jersey due to hurricane "Sandy" in October 2012, or more recently in February 2013, the power outage during Super Bowl 2013, which lasted for 34 minutes.

In coming years, power grids in the United States, Europe, and other regions worldwide are expected to undergo major paradigm shifts. Today, Internet technology and renewable energies are beginning to merge in order to create the infrastructure for the TIR economy, which has been officially endorsed by the European Commission as economic growth roadmap toward a competitive low carbon society by 2050 and has been implemented by several early adopting countries such as Germany, England, and Italy, as well as cities such as San Antonio, TX, USA, among others [103]. It has been receiving an increasing amount of attention by other key players, for example, the Government of China most recently. In the coming era, millions of consumers will produce their own renewable energy and share it with each other via an integrated and seamless Energy Internet, similar to the way we use to create and share information online nowadays.

The future Energy Internet aims at not only addressing the reliability issues of current power grids but also offering several additional major benefits. The Energy Internet will be instrumental in realizing the vision of the smart grid by incorporating sophisticated sensing, monitoring, information, and communications technologies to provide better power grid performance, engage customers to play an interactive role, and support a wide range of additional services to both utilities and consumers. Potential smart grid applications include substation and distribution automation, advanced metering infrastructure (AMI), wide-area situational awareness (WASA), home energy and demand response management, outage management, distributed generation and renewables, and grid-to-vehicle/vehicle-to-grid (G2V/V2G) electricity storage and charging applications for plug-in electric vehicles (PEVs) [104]. The authors also quantified the communications requirements of the aforementioned smart grid applications in terms of latency, bandwidth, reliability, and security and concluded that a reliable and fast smart grid communications infrastructure is necessary to enable real-time exchange of data among distributed power grid elements, for example, power generators, energy storage systems, and users.

Recently, a number of major telecommunication service providers such as KT and Telecom Italia have started to move into the energy market. An interesting example is Deutsche Telekom's new offering of virtual power plants, where homes deploy combined heat and power plants (CHPPs) on site to locally supply both hot water and power, thereby reducing the load on the power grid and avoiding transmission line losses ("Deutsche Telekom delivers virtual power plants," April 2012 (Online available at: http://www.telekom.com/ media/enterprise-solutions/121194)). Importantly, note that business models, arguably more than technological choices, play a key role in the rollout of smart grid communications infrastructures. According to [105], utilities along with municipalities are responsible for $22 \%$ of households passed with FTTB/H in Europe. These investments enable utilities and/or municipalities to (i) leverage their existing duct, sewer, and other infrastructures, (ii) create a new source of revenue in the face of ongoing liberalization of the energy sector, particularly in smart grid solutions, and (iii) provide services completely independent from incumbents' infrastructures. Furthermore, it was recently shown in [106] that cooperation 
among different utilities in the rollout phase may drive down the CAPEX of $\mathrm{FTTB} / \mathrm{H}$ deployments by $17 \%$. Innovative partnerships enable utilities and other players to share smart grid communications infrastructures investments by transitioning from the traditional vertical network integration model towards splitting the value chain into a threetier business model that consists of network infrastructure rollout, network operation/maintenance, and service provisioning [105]. One of the most promising examples of such a multitier business model is the Swiss Fibre Net of OPENAXS, an association of currently 22 regional electricity utilities throughout Switzerland (see also http:// www.openaxs.ch/). The goal of Swiss Fibre Net is to create added value for consumers by having $30 \%$ of FTTB/H connected households by 2013 and $80 \%$ by 2020 . The power utilities are responsible for the installation of the network infrastructure as well as its operation and maintenance but leave its access open to all (e.g., triple-play voice, video, and data) service providers on a nondiscriminatory basis. Another interesting example is the recent interest of Chinese utility companies, for example, State Grid Corporation of China (SGCC), in PON equipment to not only backhaul electric data on usage and outages of their power networks but also, and arguably more interestingly, to offer FTTH services to consumers and businesses ("China's smart grid drive creates $\$ 1.5$ billion opportunity for PON vendors, says Ovum," January 2013 (Online available at: http://www .fiercetelecom.com/story/chinas-smart-grid-drive-creates-15billion-opportunity-pon-vendors-says-ovu/2013-01-09)).

A plethora of wired and wireless networking technologies exists to realize smart grid communications infrastructures [107]. It is important to note, however, that in general the goal of utilities is to use only a small number of low-cost, simple, reliable, and future-proof smart grid communications technologies that remain in place for decades after installation. It is also worthwhile to mention that IEEE P2030, one of the first smart grid standards, does not specify any communications technology of choice for the future smart grid gradually evolving between now and 2030, though it is favorable to rely on the exceptionally low latency characteristics of fiber optic facilities, either owned or leased by the smart grid operator, and wireless technologies, where fiber is available to some but not all points in the system [108].

In $[109,110]$, we recently described a variety of advanced techniques to render FiWi broadband access networks dependable, including optical coding based fiber fault monitoring techniques, localized optical redundancy strategies, wireless extensions, and availability-aware routing algorithms, in order to improve their reliability, availability, and survivability as well security and safety. Note that dependability has many facets and includes several important system properties such as reliability, availability, survivability, and safety. Recall from above that beside reliability, latency is a key requirement of not only smart grid communications but also LTE-A fiber backhaul infrastructures. We refer the interested reader to [111] for more detailed information on various techniques to lower the latency of NG-PON based smart grid communications and mobile backhaul infrastructures.

\section{Conclusions}

FiWi networking research deals with the integration of optical fiber and wireless broadband access technologies. The resultant FiWi broadband access networks may deploy both centralized RoF and distributed R\&F networking technologies. Significant progress has been made in the areas of FiWi network architecture design, optimal ONU placement, direct inter-ONU communications, wireless and integrated opticalwireless routing, and end-to-end QoS support, whereby the vast majority of previous FiWi studies considered cascaded architectures consisting of a single-stage TDM or WDM PON and a multihop WMN based on legacy or VHT WLAN technologies. Recent FiWi research activities focused on the design and development of "green" network architectures as well as energy-efficient MAC and routing protocols, advanced survivability techniques, NG-PONs, and integration of fiber optic and wireless sensors. Another important development is the increasing interest of many mobile network operators in the implementation of the common public radio interface (CPRI). CPRI backhauling holds promise to provide several advantages such as reduced CAPEX/OPEX and better utilization of centralized baseband unit (BBU) processing power due to virtualization, among others. Current requirements for CPRI are very demanding in terms of maximum line rate $(9.83 \mathrm{~Gb} / \mathrm{s})$ and maximum transport delay $(<200 / 300 \mu$ s without including propagation delay). To meet these requirements, only optical fiber represents a viable option for CPRI backhaul transport. It was shown in [112] that TDM based fiber architectures can be a very cost effective CPRI backhaul solution, though future RSOA based WDM backhaul solutions may become an economically viable alternative for providing increased capacity.

We have elaborated on emerging trends that will shift the research focus of future FiWi broadband access networks to their deployment across other relevant economic sectors in response to the current global economic crisis, leading markets and society to the next golden age, where the benefits of new technologies are fully realized across the entire economy by offering a vast innovation and growth potential across multiple economic sectors. It is expected that capacity-centric FiWi access networks and coverage-centric mobile networks will converge to give rise to unified FiWi/LTE-A HetNets, whereby the sharing of existent high-capacity FTTx infrastructures with low-latency small-cell backhaul capability and using the WLAN mesh front-end for WiFi offloading of rapidly increasing mobile data traffic from cellular networks will have a highly beneficial impact on their performance and cost. Low-latency, low-cost, and reliable FiWi access networks will also play a key role in realizing future smart grid communications infrastructures to facilitate the convergence of Internet technology and renewable energies via an integrated and seamless Energy Internet and to usher in a sustainable Third Industrial Revolution economy.

\section{Conflict of Interests}

The author declares that there is no conflict of interests regarding the publication of this paper. 


\section{References}

[1] OECD, "Broadband and the economy," Ministerial Background Report DSTI/ICCP/IE(2007)3/FINAL, OECD, Seoul, Republic of Korea, 2007.

[2] OECD, "OECD Broadband Portal," http://www.oecd.org/sti/ ict/broadband.

[3] T. Koonen, "Fiber to the home/fiber to the premises: what, where, and when?" Proceedings of the IEEE, vol. 94, no. 5, pp. 911-934, 2006.

[4] Ericsson, "Deep fiber access," White Paper, 2008.

[5] P. Ödling, T. Magesacher, S. Host, P. O. Börjesson, M. Berg, and E. Areizaga, "The fourth generation broadband concept," IEEE Communications Magazine, vol. 47, no. 1, pp. 63-69, 2009.

[6] W. Fischer, "Point-to-point FTTx," in Broadband Access Networks: Technologies and Deployments, A. Shami, M. Maier, and C. Assi, Eds., Springer, New York, NY, USA, 2009.

[7] F. Effenberger, D. Cleary, O. Haran et al., "An introduction to PON technologies," IEEE Communications Magazine, vol. 45, no. 3, pp. S17-S25, 2007.

[8] M. Maier, N. Ghazisaidi, and M. Reisslein, "The audacity of fiber-wireless (FiWi) networks," in Proceedings of the 3rd International Conference on Access Networks (AccessNets '08), pp. 110, October 2008.

[9] S. Aissa and M. Maier, "Towards seamless fiber-wireless (FiWi) access networks: convergence and challenges," in Proceedings of the International Conference on Transparent Optical Networks "Mediterranean Winter" (ICTON-MW'07), pp. 1-6, December 2007.

[10] J. MacLeod and S. R. Safavian, "FMC: fixed-mobile convergence," Bechtel Technology Journal, vol. 1, no. 1, pp. 57-75, 2008.

[11] F. G. Harrison and S. R. Hearnden, "Challenge to realize convergence of fixed and mobile communications," Electronics \& Communication Engineering Journal, vol. 11, no. 3, pp. 164-168, 1999.

[12] Y. Luo, T. Wang, S. B. Weinstein, M. Cvijetic, and S. Nakamura, "Integrating optical and wireless services in the access network," in Proceedings of the Optical Fiber Communication/National Fiber Optic Engineers Conference (OFC/NFOEC '06), March 2006.

[13] C. C. Davis, I. I. Smolyaninov, and S. D. Milner, "Flexible optical wireless links and networks," IEEE Communications Magazine, vol. 41, no. 3, pp. 51-57, 2003.

[14] T.-S. Chu and M. J. Gans, "Fiber optic microcellular radio," IEEE Transactions on Vehicular Technology, vol. 40, no. 3, pp. 599606, 1991.

[15] B. L. Dang and I. Niemegeers, "Analysis of IEEE 802.11 in radio over fiber home networks," in Proceedings of the IEEE Conference on Local Computer Networks (LCN '05), pp. 744-747, November 2005.

[16] P. S. Henry, "Integrated optical/wireless alternatives for the metropolitan environment," IEEE Communications Society Webinar, 2007.

[17] Z. Jia, J. Yu, G. Ellinas, and G.-K. Chang, "Key enabling technologies for optical wireless networks: optical millimeter-wave generation, wavelength reuse, and architecture," IEEE/OSA Journal of Lightwave Technology, vol. 25, no. 11, pp. 3452-3471, 2007.

[18] S. Sarkar, S. Dixit, and B. Mukherjee, "Hybrid wireless-optical broadband-access network (WOBAN): a review of relevant challenges," IEEE/OSA Journal of Lightwave Technology, vol. 25, no. 11, pp. 3329-3340, 2007.
[19] N. Ghazisaidi and M. Maier, "Hierarchical frame aggregation techniques for hybrid fiber-wireless access networks," IEEE Communications Magazine, vol. 49, no. 9, pp. 64-73, 2011.

[20] B. Lannoo, D. Colle, M. Pickavet, and P. Demeester, "Radioover-fiber-based solution to provide broadband internet access to train passengers," IEEE Communications Magazine, vol. 45, no. 2, pp. 56-62, 2007.

[21] N. Pleros, K. Vyrsokinos, K. Tsagkaris, and N. D. Tselikas, "A $60 \mathrm{GHz}$ radio-over-fiber network architecture for seamless communication with high mobility," IEEE/OSA Journal of Lightwave Technology, vol. 27, no. 12, pp. 1957-1967, 2009.

[22] A. Das, A. Nkansah, N. J. Gomes, I. J. Garcia, J. C. Batchelor, and D. Wake, "Design of low-cost multimode fiber-fed indoor wireless networks," IEEE Transactions on Microwave Theory and Techniques, vol. 54, no. 8, pp. 3426-3432, 2006.

[23] G. Shen, R. S. Tucker, and C.-J. Chae, "Fixed mobile convergence architectures for broadband access: integration of EPON and WiMAX," IEEE Communications Magazine, vol. 45, no. 8, pp. 44-50, 2007.

[24] V. Muralidharan, A. M. Wyglinski, and W. Wong, "HiFi-WiN: hybrid integrated fiber-wireless networking for broadband metropolitan area access," in Proceedings of the Virginia Tech Symposium on Wireless Personal Communications, pp. 1-8, June 2007.

[25] W.-P. Lin, M.-S. Kao, and S. Chi, "A reliable architecture for broad-band fiber-wireless access networks," IEEE Photonics Technology Letters, vol. 15, no. 2, pp. 344-346, 2003.

[26] S. Bhandari and E. K. Park, "Hybrid optical wireless networks," in Proceedings of the International Conference on Networking, International Conference on Systems and International Conference on Mobile Communications and Learning Technologies (ICN/ICONS/MCL '06), pp. 1-5, April 2006.

[27] W.-T. Shaw, S.-W. Wong, N. Cheng et al., "Hybrid architecture and integrated routing in a scalable optical-wireless access network," IEEE/OSA Journal of Lightwave Technology, vol. 25, no. 11, pp. 3443-3451, 2007.

[28] M. Maier, M. Lévesque, and L. Ivanescu, "NG-PONs 1\&2 and beyond: the dawn of the uber-FiWi network," IEEE Network, vol. 26, no. 2, pp. 15-21, 2012.

[29] M. D. Andrade, G. Kramer, L. Wosinska, J. Chen, S. Sallent, and B. Mukherjee, "Evaluating strategies for evolution of passive optical networks," IEEE Communications Magazine, vol. 49, no. 7, pp. 176-184, 2011.

[30] G. Kramer, M. D. Andrade, R. Roy, and P. Chowdhury, "Evolution of optical access networks: architectures and capacity upgrades," Proceedings of the IEEE, vol. 100, no. 5, pp. 1188-1196, 2012.

[31] W. T. Shaw, S. W. Wong, S. H. Yen, and L. G. Kazovsky, "An ultra-scalable broadband architecture for municipal hybrid wireless access using optical grid network," in Proceedings of the Optical Fiber Communication/National Fiber Optic Engineers Conferencem (OFC/NFOEC '09), pp. 1-3, March 2009.

[32] S.-W. Wong, D. R. Campelo, N. Cheng et al., "Grid reconfigurable optical-wireless architecture for large scale municipal mesh access network," in Proceedings of the IEEE Global Telecommunications Conference (GLOBECOM '09), pp. 1-6, December 2009.

[33] F. Diehm, P. Marsch, and G. Fettweis, “The FUTON prototype: proof of concept for coordinated multi-point in conjunction with a novel integrated wireless/optical architecture," in Proceedings of the IEEE Wireless Communications and Networking Conference Workshops (WCNCW'10), pp. 1-4, April 2010. 
[34] F. Diehm, J. Holfeld, G. Fettweis et al., "The FUTON prototype: broadband communication through coordinated multipoint using a novel integrated optical/wireless architecture," in Proceedings of the IEEE GLOBECOM Workshops (GC '10), pp. 757-762, December 2010.

[35] P. Monteiro, S. Pato, E. López, D. Wake, N. J. Gomes, and A. Gameiro, "Fiber optic networks for distributed radio architectures: FUTON concept and operation," in Proceedings of the IEEE Wireless Communications and Networking Conference Workshops (WCNCW '10), pp. 1-5, April 2010.

[36] K. Kanonakis, I. Tomkos, T. Pfeiffer, J. Prat, and P. Kourtessis, "ACCORDANCE: a novel OFDMA-PON paradigm for ultrahigh capacity converged wireline-wireless access networks," in Proceedings of the 12th International Conference on Transparent Optical Networks (ICTON '10), pp. 1-4, July 2010.

[37] M. Milosavljevic, P. Kourtessis, and J. M. Senior, "Transparent wireless transmission over the ACCORDANCE optical/wireless segment," in Proceedings of the 7th International Symposium on Communication Systems, Networks and Digital Signal Processing (CSNDSP '10), pp. 138-142, July 2010.

[38] K. Kanonakis, I. Tomkos, H.-G. Krimmel et al., "Results from the EU project ACCORDANCE on converged OFDMA-PON networks," in Proceedings of the 15th International Conference on Transparent Optical Networks( ICTON '13), pp. 1-4, July 2013.

[39] S. Sarkar, H.-H. Yen, S. Dixit, and B. Mukherjee, "A novel delayaware routing algorithm (DARA) for a hybrid wireless-optical broadband access network (WOBAN)," IEEE Network, vol. 22, no. 3, pp. 20-28, 2008.

[40] S. Sarkar, H.-H. Yen, S. Dixit, and B. Mukherjee, "RADAR: risk-and-delay aware routing algorithm in a hybrid wirelessoptical broadband access network (WOBAN)," in Proceedings of the Optical Fiber Communication and the National Fiber Optic Engineers Conference (OFC/NFOEC '07), pp. 1-3, March 2007.

[41] S. Sarkar, H.-H. Yen, S. Dixit, and B. Mukherjee, "Hybrid wireless-optical broadband access network (WOBAN): network planning using Lagrangean relaxation," IEEE/ACM Transactions on Networking, vol. 17, no. 4, pp. 1094-1105, 2009.

[42] Z. Zheng, J. Wang, and X. Wang, "ONU placement in fiberwireless (FiWi) networks considering peer-to-peer communications," in Proceedings of the IEEE Global Telecommunications Conference (GLOBECOM '09), pp. 1-7, December 2009.

[43] Z. Zehng, J. Wang, and J. Wang, "A study of network throughput gain in optical-wireless (FiWi) networks subject to peer-topeer communications," in Proceedings of the IEEE International Conference on Communications (ICC '09), pp. 1-6, June 2009.

[44] Y. Li, J. Wang, C. Qiao, A. Gumaste, Y. Xu, and Y. Xu, "Integrated fiber-wireless (FiWi) access networks supporting inter-ONU communications," IEEE/OSA Journal of Lightwave Technology, vol. 28, no. 5, pp. 714-724, 2010.

[45] W.-T. Shaw, S.-W. Wong, N. Cheng et al., "Reconfigurable optical backhaul and integrated routing algorithm for load balancing in hybrid optical-wireless access networks," in Proceedings of the IEEE International Conference on Communications (ICC '08), pp. 5697-5701, May 2008.

[46] X. Chen, R. Abu, L. Shi et al., "Delay-differentiated routing algorithm to enhance delay performance of WOBAN," in Proceedings of the 9th International Conference on Optical Internet (COIN '10), pp. 1-4, July 2010.

[47] A. Reaz, V. Ramamurthi, and S. Sarkar, "Capacity and delay aware routing in hybrid wireless-optical broadband access network," in Proceedings of the 1st International Symposium on
Advanced Networks and Telecommunications Systems (ANTS '07), pp. 1-2, December 2007.

[48] A. Reaz, V. Ramamurthi, and S. Sarkar, "Flow-aware channel assignment for multi-radio wireless-optical broadband access network," in Proceedings of the 2nd International Symposium on Advanced Networks and Telecommunication Systems (ANTS '08), pp. 1-3, December 2008.

[49] A. Reaz, V. Ramamurthi, S. Sarkar, D. Ghosal, S. Dixit, and B. Mukherjee, "CaDAR: an efficient routing algorithm for wireless-optical broadband access network," in Proceedings of the IEEE International Conference on Communications (ICC '08), pp. 5191-5195, May 2008.

[50] A. Reaz, V. Ramamurthi, S. Sarkar, D. Ghosal, and B. Mukherjee, "Hybrid wireless-optical broadband access network (WOBAN): capacity enhancement for wireless access," in Proceedings of the IEEE Global Telecommunications Conference (GLOBECOM '08), pp. 1-5, December 2008.

[51] A. Reaz, V. Ramamurthi, S. Sarkar, D. Ghosal, S. Dixit, and B. Mukherjee, "CaDAR: an efficient routing algorithm for a wirelessoptical broadband access network (WOBAN)," IEEE/OSA Journal of Optical Communications and Networking, vol. 1, no. 5, pp. 392-403, 2009.

[52] X. Shao, Y. K. Yeo, L. H. Ngoh, X. Cheng, W. Rong, and L. Zhou, "Availability-aware routing for large-scale hybrid wirelessoptical broadband access network," in Proceedings of the Conference on Optical Fiber Communication, Collocated National Fiber Optic Engineers Conference (OFC/NFOEC '10), pp. 1-3, March 2010.

[53] M. Kiese, E. Georgieva, D. Schupke, B. Mukherjee, and J. Eberspächer, "Availability evaluation in hybrid wireless-optical broadband access networks," in Proceedings of the IEEE International Conference on Communications (ICC '09), pp. 1-6, June 2009.

[54] J. Wang, K. Wu, S. Li, and C. Qiao, "Performance modeling and analysis of multi-path routing in integrated fiber-wireless networks," in Proceedings of the 29th IEEE Conference on Computer Communications (INFOCOM '10), pp. 1-5, March 2010.

[55] R. S. Tucker, R. Parthiban, J. Baliga, K. Hinton, R. W. A. Ayre, and W. V. Sorin, "Evolution of WDM optical IP networks: a cost and energy perspective," IEEE/OSA Journal of Lightwave Technology, vol. 27, no. 3, pp. 243-252, 2009.

[56] B. Mukherjee and P. Chowdhury, "Green wireless-optical broadband access network (WOBAN)," in Proceedings of the OSA Asia Communications and Photonics Conference and Exhibition (ACP '09), pp. 1-2, November 2009.

[57] P. Chowdhury, B. Mukherjee, S. Sarkar, G. Kramer, and S. Dixit, "Hybrid wireless-optical broadband access network(WOBAN): prototype development and research challenges," IEEE Network, vol. 23, no. 3, pp. 41-48, 2009.

[58] P. Chowdhury, M. Tornatore, S. Sarkar, and B. Mukherjee, "Building a green wireless-optical broadband access network (WOBAN)," IEEE/OSA Journal of Lightwave Technology, vol. 28, no. 16, pp. 2219-2229, 2010.

[59] M. Maier and N. Ghazisaidi, "QoS provisioning techniques for future fiber-wireless (FiWi) access networks," Future Internet, vol. 2, no. 2, pp. 126-155, 2010.

[60] Y. Luo, S. Yin, T. Wang et al., "QoS-aware scheduling over hybrid optical wireless networks," in Proceedings of the Optical Fiber Communication and the National Fiber Optic Engineers Conference (OFC/NFOEC '07), pp. 1-7, March 2007.

[61] Y. Yan, H. Yu, H. Wessing, and L. Dittmann, "Enhanced signaling scheme with admission control in the hybrid optical wireless 
(HOW) networks," in Proceedings of the IEEE INFOCOM Workshops, pp. 1-6, April 2009.

[62] J. Lee, S. H. S. Newaz, J. K. Choi, G. M. Lee, and N. Crespi, "QoS mapping over hybrid optical and wireless access networks," in Proceedings of the 1st International Conference on Evolving Internet (INTERNET '09), pp. 139-141, August 2009.

[63] A. Belbekkouche, J. Rezgui, and A. Hafid, "QoS provisioning for wireless mesh and optical burst switching convergence," in Proceedings of the IEEE Wireless Communications and Networking Conference (WCNC '10), pp. 1-6, April 2010.

[64] A. Belbekkouche, J. Rezgui, and A. Hafid, "Wireless mesh and optical burst switching convergence for a novel metropolitan area network architecture," Computer Networks, vol. 55, no. 1, pp. 159-172, 2011.

[65] J. Rezgui, A. Belbekkouche, and A. Hafid, "On delay performance and burst assembly for wireless mesh and optical burst switching converged metro area network," Mobile Networks and Applications, vol. 16, no. 1, pp. 122-133, 2011.

[66] Z. M. Fadlullah, H. Nishiyama, N. Kato, H. Ujikawa, K.-I. Suzuki, and N. Yoshimoto, "Smart FiWi networks: challenges and solutions for QoS and green communications," IEEE Intelligent Systems, vol. 28, no. 2, pp. 86-91, 2013.

[67] B. Kantarci and H. T. Mouftah, "Energy efficiency in the extended-reach fiber-wireless access networks," IEEE Network, vol. 26, no. 2, pp. 28-35, 2012.

[68] X. Liu, N. Ghazisaidi, L. Ivanescu, R. Kang, and M. Maier, "On the tradeoff between energy saving and Qos support for video delivery in EEE-based FiWi networks using real-world traffic traces," IEEE/OSA Journal of Lightwave Technology, vol. 29, no. 18, pp. 2670-2676, 2011.

[69] M. Lévesque, M. Maier, F. Aurzada, and M. Reisslein, "Analytical framework for the capacity and delay evaluation of nextgeneration FiWi network routing algorithms," in Proceedings of the IEEE Wireless Communications and Networking Conference (WCNC '13), pp. 1926-1931, April 2013.

[70] F. Aurzada, M. Lévesque, M. Maier, and M. Reisslein, "FiWi access networks based on next-generation PON and gigabitclass WLAN technologies: a capacity and delay analysis," IEEE/ACM Transactions on Networking, 2013.

[71] N. Ghazisaidi, M. Scheutzow, and M. Maier, "Survivability analysis of next-generation passive optical networks and fiberwireless access networks," IEEE Transactions on Reliability, vol. 60, no. 2, pp. 479-492, 2011.

[72] M. Maier, "Survivability techniques for NG-PONs and FiWi access networks," in Proceedings of the IEEE International Conference on Communications (ICC '12), pp. 6214-6219, June 2012.

[73] A. Attar, H. Li, V. C. M. Leung, and Q. Pang, "Cognitive wireless local area network over fibers: architecture, research issues and testbed implementation," IEEE Communications Magazine, vol. 50, no. 6, pp. 107-113, 2012.

[74] M. Ahmed, D. Habibi, and I. Ahmad, "An improved architecture for integrating fourth generation wireless and passive optical networks," in Proceedings of the IEEE Region 10 Conference: Sustainable Development through Humanitarian Technology (TENCON '12), pp. 1-6, November 2012.

[75] X. Liu, K. Fouli, R. Kang, and M. Maier, "Network-codingbased energy management for next-generation passive optical networks," IEEE/OSA Journal of Lightwave Technology, vol. 30, no. 6, pp. 864-875, 2012.

[76] F. Aurzada, M. Scheutzow, M. Reisslein, N. Ghazisaidi, and M. Maier, "Capacity and delay analysis of next-generation passive optical networks (NG-PONs)," IEEE Transactions on Communications, vol. 59, no. 5, pp. 1378-1388, 2011.

[77] M. S. Kiaei, C. Assi, L. Meng, and M. Maier, "On the coexistence of 10G-EPONs and WDM-PONs: a scheduling and bandwidth allocation approach," IEEE/OSA Journal of Lightwave Technology, vol. 29, no. 10, pp. 1417-1426, 2011.

[78] M. Kiaei, K. Fouli, M. Scheutzow, M. Maier, M. Reisslein, and C. Assi, "Low-latency polling schemes for long-reach passive optical networks," IEEE Transactions on Communications, vol. 61, no. 7, pp. 2936-2945, 2013.

[79] M. S. Kiaei, K. Fouli, M. Scheutzow, M. Maier, M. Reisslein, and C. Assi, "Delay analysis for Ethernet long-reach passive optical networks," in Proceedings of the IEEE International Conference on Communications (ICC '12), pp. 3099-3104, June 2012.

[80] R. Llorente, M. Morant, F. Martinez et al., "Impairment compensation in long-reach integrated optical-wireless PON," in Proceedings of the 21st Future Network \& Mobile Summit (FutureNetw '12), pp. 1-9, July 2012.

[81] N. Ghazisaidi, M. Maier, and M. Reisslein, "VMP: a MAC protocol for EPON-based video-dominated FiWi access networks," IEEE Transactions on Broadcasting, vol. 58, no. 3, pp. 440-453, 2012.

[82] L. Yu, H. Liu, S. Hu, and Y. Zhang, "ONUs placement for reduced sensors' energy consumption in PON-WSN hybrid networks," in Proceedings of the 7th International ICST Conference on Communications and Networking in China (CHINACOM '12), pp. 457-462, August 2012.

[83] M. Hossen and M. Hanawa, "Adaptive limited DBA algorithm for multi-OLT PON-based FTTH and wireless sensor netwroks," in Proceedings of the 18th Asia-Pacific Conference on Communications: "Green and Smart Communications for IT Innovation” (APCC '12), pp. 372-377, October 2012.

[84] Y. Tanaka, M. Kinoshita, and T. Kurokawa, "Laser-driven lowpower fiber sensor network integrated with wireless sensors," in Proceedings of the Conference on Lasers and Electro-Optics (CLEO '12), pp. 1-2, May 2012.

[85] T. Kuri, H. Harai, N. Wada, T. Kawanishi, and M. Hosokawa, "Adaptable access system: pursuit of ideal future access system architecture," IEEE Network, vol. 26, no. 2, pp. 42-48, 2012.

[86] J. G. Andrews, "Seven ways that HetNets are a cellular paradigm shift," IEEE Communications Magazine, vol. 51, no. 3, pp. 136144, 2013.

[87] T. Biermann, L. Scalia, C. Choi, W. Kellerer, and H. Karl, "How backhaul networks influence the feasibility of coordinated multipoint in cellular networks," IEEE Communications Magazine, vol. 51, no. 8, pp. 168-176, 2013.

[88] C. Perez, "Unleashing a golden age after the financial collapse: drawing lessons from history," Environmental Innovation and Societal Transitions, vol. 6, pp. 9-23, 2013.

[89] J. G. Andrews, H. Claussen, M. Dohler, S. Rangan, and M. C. Reed, "Femtocells: past, present, and future," IEEE Journal on Selected Areas in Communications, vol. 30, no. 3, pp. 497-508, 2012.

[90] J. Hoydis, M. Kobayashi, and M. Debbah, "Green small-cell networks," IEEE Vehicular Technology Magazine, vol. 6, no. 1, pp. 37-43, 2011.

[91] P. Bhat, S. Nagata, L. Campoy et al., "LTE-advanced: an operator perspective," IEEE Communications Magazine, vol. 50, no. 2, pp. 104-114, 2012. 
[92] K. I. Pedersen, P. H. Michaelsen, C. Rosa, and S. Barbera, "Mobility enhancements for LTE-advanced multilayer networks with inter-site carrier aggregation," IEEE Communications Magazine, vol. 51, no. 5, pp. 64-71, 2013.

[93] P. Muñoz, R. Barco, D. Laselva, and P. Mogensen, "Mobilitybased strategies for traffic steering in heterogeneous networks," IEEE Communications Magazine, vol. 51, no. 5, pp. 54-62, 2013.

[94] M. Peng, D. Liang, Y. Wei, J. Li, and H.-H. Chen, "Self-configuration and self-optimization in LTE-advanced heterogeneous networks," IEEE Communications Magazine, vol. 51, no. 5, pp. 36-45, 2013.

[95] R. Barco, P. Lazaro, and P. Munoz, "A unified framework for selfhealing in wireless networks," IEEE Communications Magazine, vol. 50, no. 12, pp. 134-142, 2012.

[96] M. Bennis, M. Simsek, A. Czylwik, W. Saad, S. Valentin, and M. Debbah, "When cellular meets WiFi in wireless small cell networks," IEEE Communications Magazine, vol. 51, no. 6, pp. 44-50, 2013.

[97] S. Singh, H. S. Dhillon, and J. G. Andrews, "Offloading in heterogeneous networks: modeling, analysis, and design insights," IEEE Transactions on Wireless Communications, vol. 12, no. 5, pp. 2484-2497, 2013.

[98] K. Lee, J. Lee, Y. Yi, I. Rhee, and S. Chong, "Mobile data offloading: how much can WiFi deliver?" IEEE/ACM Transactions on Networking, vol. 21, no. 2, pp. 536-550, 2013.

[99] J. Zhang, L. Shan, H. Hu, and Y. Yang, "Mobile cellular networks and wireless sensor networks: toward convergence," IEEE Communications Magazine, vol. 50, no. 3, pp. 164-169, 2012.

[100] H. Whu, C. Zhu, R. J. La, X. Liu, and Y. Zhang, "FASA: accelerated S-ALOHA using access history for event-driven M2M communications," IEEE/ACM Transactions on Networking, vol. 21, no. 6, pp. 1904-1917, 2013.

[101] OECD, "Machine-to-machine communications: connecting billions of devices," OECD Digital Economy Papers no. 192, OECD, Seoul, Republic of Korea, 2012.

[102] R. Kubo, M. Tadokoro, T. Kubo, K. Suzuki, and N. Yoshimoto, "Performance evaluation of energy-efficient PONs for largescale sensor-actuator networks," in Proceedings of the IEEE International Conference on Mechatronics (ICM), pp. 898-903, Vicenza, Italy, March 2013.

[103] J. Rifkin, "Beyond austerity a sustainable third industrial revolution economic growth plan for the European Union," in Proceedings of the Mission Growth Summit: Europe at the Lead of the New Industrial Revolution, The European Commission, May 2012.

[104] V. C. Gungor, D. Sahin, T. Kocak et al., "A survey on smart grid potential applications and communication requirements," IEEE Transactions on Industrial Informatics, vol. 9, no. 1, pp. 28-42, 2013.

[105] D. Arthur Little, "FTTH: double squeeze of incumbentsforced to partner?" 2010.

[106] M. Tahon, J. V. Ooteghem, K. Casier et al., "Cost allocation model for a synergetic cooperation in the rollout of telecom and utility networks," in Proceedings of the Conference on Telecommunications Internet and Media Techno-Economics (CTTE '11), pp. 1-7, May 2011.

[107] X. Fang, S. Misra, G. Xue, and D. Yang, "Smart grid-the new and improved power grid: a survey," IEEE Communications Surveys \& Tutorials, vol. 14, no. 4, pp. 944-980, 2012.
[108] V. K. Sood, D. Fischer, J. M. Eklund, and T. Brown, "Developing a communication infrastructure for the smart grid," in Proceedings of the IEEE Electrical Power \& Energy Conference (EPEC '13), pp. 119-130, March 2013.

[109] M. Maier, "Reliable fiber-wireless access networks: less an end than a means to an end," in Proceedings of the 9th International Conference on the Design of Reliable Communication Networks (DRCN '09), pp. 1-7, October 2009.

[110] M. Maier and M. Lévesque, "Dependable fiber-wireless (FiWi) access networks and their role in a sustainable third industrial revolution economy," IEEE Transactions on Reliability, vol. 63, no. 2, pp. 386-400, 2014.

[111] M. Maier, "The escape of sisyphus or what "Post NG-PON2" should do for a sustainable TIR economy apart from neverending capacity upgrades," Photonics, vol. 1, no. 1, pp. 47-66, 2014.

[112] A. Lometti, "Optical access architectures for backhauling of broadband mobile networks," in Proceedings of the 15th International Telecommunications Network Strategy and Planning Symposium (NETWORKS '12), pp. 1-6, October 2012. 

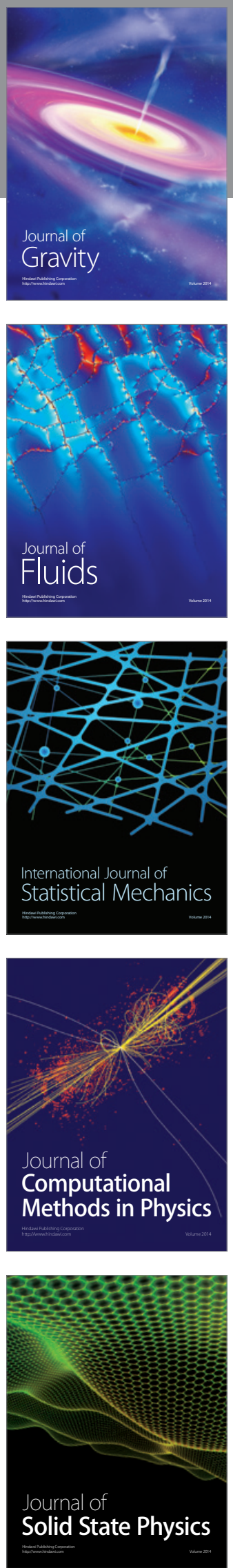

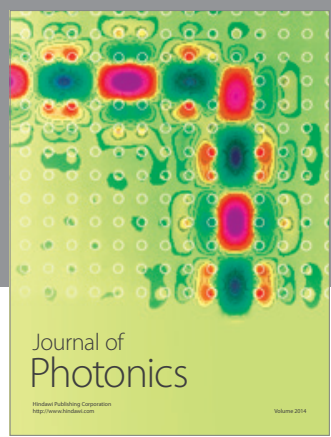

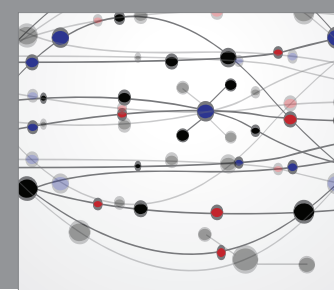

The Scientific World Journal

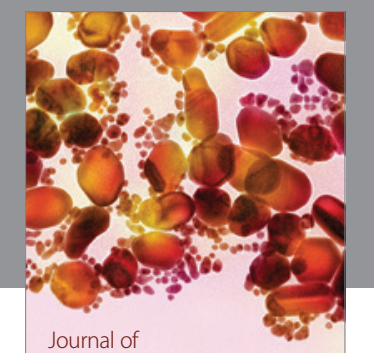

Soft Matter
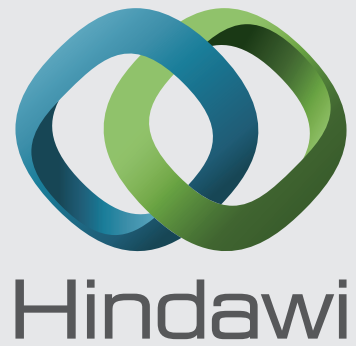

Submit your manuscripts at

http://www.hindawi.com
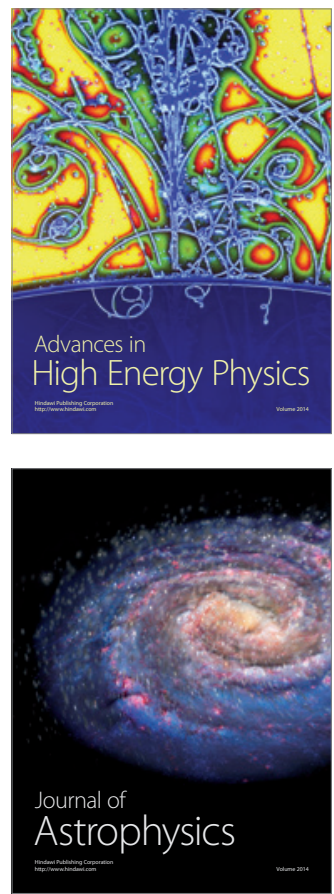
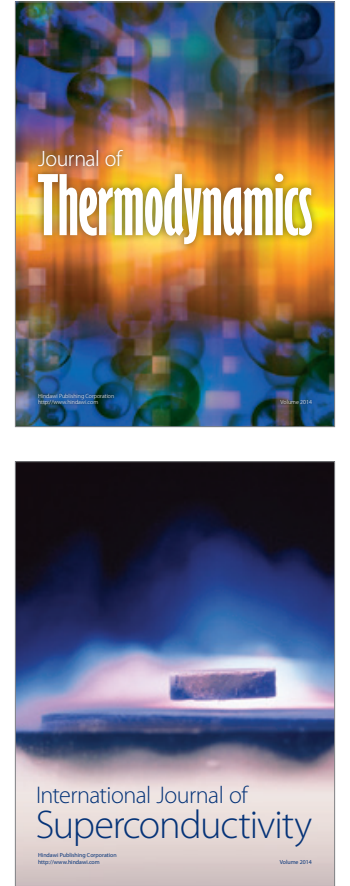
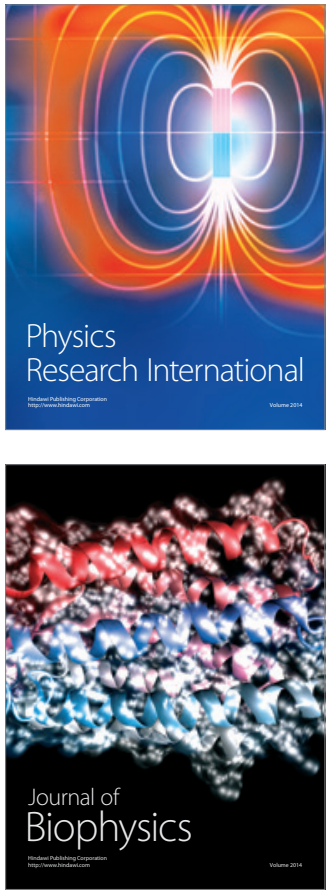
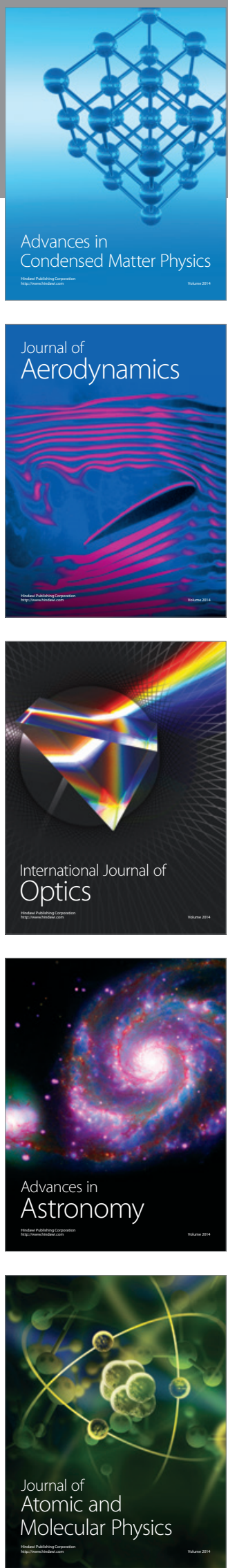